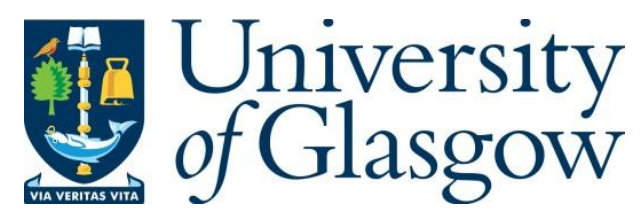

Li, X. (2018) Continuous orbit equivalence rigidity. Ergodic Theory and Dynamical Systems, 38(4), pp. 1543-1563.

There may be differences between this version and the published version. You are advised to consult the publisher's version if you wish to cite from it.

http://eprints.gla.ac.uk/210207/

Deposited on: 4 March 2020

Enlighten - Research publications by members of the University of Glasgow http://eprints.gla.ac.uk 


\title{
CONTINUOUS ORBIT EQUIVALENCE RIGIDITY
}

\author{
XIN LI
}

\begin{abstract}
We take the first steps towards a better understanding of continuous orbit equivalence, i.e., topological orbit equivalence with continuous cocycles. First, we characterise continuous orbit equivalence in terms of isomorphisms of $\mathrm{C}^{*}$-crossed products preserving Cartan subalgebras. This is the topological analogue of the classical result by Singer and FeldmanMoore in the measurable setting. Secondly, we turn to continuous orbit equivalence rigidity, i.e., the question whether for certain classes of topological dynamical systems, continuous orbit equivalence implies conjugacy. We show that this is not always the case by constructing topological dynamical systems (actions of free abelian groups, and also non-abelian free groups) which are continuously orbit equivalent but not conjugate. Furthermore, we prove positive rigidity results. For instance, it turns out that general topological Bernoulli actions are rigid when compared with actions of nilpotent groups, and that topological Bernoulli actions of duality groups are rigid when compared with actions of solvable groups. The same is true for certain subshifts of full shifts over finite alphabets.
\end{abstract}

\section{INTRODUCTION}

From its very beginning on, the theory of operator algebras was closely related to ergodic theory and dynamical systems. The bridge between these subjects is built by crossed product constructions, attaching von Neumann algebras to measure-preserving dynamical systems and $\mathrm{C}^{*}$-algebras to topological dynamical systems.

In the setting of von Neumann algebras, the crossed product construction, also called groupmeasure space construction, played an important role in the classification of injective factors. Similarly, in the $\mathrm{C}^{*}$-algebraic setting, crossed products attached to topological dynamical systems provide interesting examples which are challenging to classify and lead to new insights.

If we want to further develop the relationship between operator algebras and dynamical systems, the following question will be crucial:

How much information do these crossed product constructions contain about the underlying dynamical systems?

It turns out that the crossed product itself might contain very little information, but if we consider the crossed product together with a commutative subalgebra (which is canonically given), then our question can be answered in a systematic way.

2010 Mathematics Subject Classification. Primary 37B05; Secondary 37A20, 46L05.

Research supported by EPSRC grant EP/M009718/1. 
To explain this in the measurable and von Neumann algebraic setting, let $G \curvearrowright X$ and $H \curvearrowright Y$ be probability measure preserving actions. Here, our measure spaces are standard, our groups are discrete and countable, and they act by Borel automorphisms. We say that $G \curvearrowright X$ and $H \curvearrowright Y$ are orbit equivalent if there exists an isomorphism of measure spaces $\varphi: X \rightarrow Y$ with $\varphi(G . x)=H . \varphi(x)$ for a.e. $x \in X$. Moreover, we let $G \ltimes X$ and $H \ltimes Y$ be the measured transformation groupoids attached to $G \curvearrowright X$ and $H \curvearrowright Y$. If our actions are (essentially) free, then $G \ltimes X$ and $H \ltimes Y$ are nothing else but the orbit equivalence relations $R(G \curvearrowright X)$ and $R(H \curvearrowright Y)$ viewed as measured groupoids. Here is a classical result:

Theorem 1.1 ([24, [5, 6]). Let $G \curvearrowright X$ and $H \curvearrowright Y$ be (essentially) free probability measure preserving actions. The following are equivalent:

- $G \curvearrowright X$ and $H \curvearrowright Y$ are orbit equivalent;

- $G \ltimes X$ and $H \ltimes Y$ are isomorphic as measured groupoids (or equivalence relations);

- there is a vN-isomorphism $\Phi: L^{\infty}(X) \rtimes G \stackrel{\cong}{\longrightarrow} L^{\infty}(Y) \rtimes H$ with $\Phi\left(L^{\infty}(X)\right)=L^{\infty}(Y)$.

The interested reader may consult [26, 8, 27] for more details.

Our first result carries over Theorem 1.1 to the topological setting. Let $G \curvearrowright X$ and $H \curvearrowright Y$ be topological dynamical systems. This means that $G$ and $H$ are countable discrete groups acting by homeomorphisms on locally compact Hausdorff spaces $X$ and $Y$.

We say that $G \curvearrowright X$ and $H \curvearrowright Y$ are continuously orbit equivalent if there exists a homeomorphism $\varphi: X \stackrel{\cong}{\longrightarrow} Y$ with inverse $\psi=\varphi^{-1}: Y \stackrel{\cong}{\longrightarrow} X$ and continuous maps $a: G \times X \rightarrow H$, $b: H \times Y \rightarrow G$ such that $\varphi(g . x)=a(g, x) . \varphi(x)$ and $\psi(h . y)=b(h, y) . \psi(y)$ for all $g \in G, x \in X$, $h \in H$ and $y \in Y$. Note that $G$ and $H$ carry the discrete topology. This notion of continuous orbit equivalence has been studied in special cases (see [11, 1, 25]), but not - at least to the best of the author's knowledge - in the general setting. There is also a weaker notion of (topological) orbit equivalence which has been studied intensively for $\mathbb{Z}^{n}$-actions on the Cantor set in the remarkable papers [11, 9, 10].

Moreover, let $G \ltimes X$ and $H \ltimes Y$ be the transformation groupoids attached to $G \curvearrowright X$ and $H \curvearrowright Y$. Here is the topological analogue of Theorem 1.1:

Theorem 1.2. Let $G \curvearrowright X$ and $H \curvearrowright Y$ be topologically free systems, and assume that $X$ and $Y$ are second countable. The following are equivalent:

- $G \curvearrowright X$ and $H \curvearrowright Y$ are continuously orbit equivalent;

- $G \ltimes X$ and $H \ltimes Y$ are isomorphic as topological groupoids;

- there is a $C^{*}$-isomorphism $\Phi: C_{0}(X) \rtimes_{r} G \stackrel{\cong}{\longrightarrow} C_{0}(Y) \rtimes_{r} H$ with $\Phi\left(C_{0}(X)\right)=C_{0}(Y)$.

Here and in the sequel, "topologically free system" stands for "topologically free topological dynamical system". Because of Theorem 1.2, it seems that - at least for our purposes - continuous orbit equivalence is a good topological analogue of orbit equivalence in the measurable setting. 
In the measurable setting, orbit equivalence rigidity has established itself as a key notion. The idea is to find classes of actions for which orbit equivalence already implies conjugacy. Indeed, impressive orbit equivalence rigidity results have been obtained in [28, 17, 15, 16, 17, 14, 13. Viewing continuous orbit equivalence as the topological analogue of orbit equivalence, a natural question is whether there are rigidity phenomena for continuous orbit equivalence.

The only result known in this context is due to [1], which says that if $\mathbb{Z} \curvearrowright X$ and $\mathbb{Z} \curvearrowright Y$ are topologically free systems on compact spaces $X$ and $Y$ such that one of them is topologically transitive, then $\mathbb{Z} \curvearrowright X$ and $\mathbb{Z} \curvearrowright Y$ must already be conjugate if they are continuously orbit equivalent. Apart from this, not much else seems to be known about continuous orbit equivalence rigidity.

The main goal of the present paper is to take the first steps towards a better understanding of continuous orbit equivalence rigidity.

First of all, we construct examples of topological dynamical systems which are continuously orbit equivalent but not conjugate. This ensures that the comparison between continuous orbit equivalence and conjugacy is really interesting. A first class of examples is given by products of odometer actions. A second family of examples is constructed from boundary actions of non-abelian free groups and odometer actions, inspired by [25].

Secondly, we prove positive results in continuous orbit equivalence rigidity.

Theorem 1.3. Let $G$ be a torsion-free group, and let $H$ be a finitely generated nilpotent group which is not virtually infinite cyclic. Assume that $G \curvearrowright X$ is a topologically free system on a compact space $X$ such that $G \curvearrowright X$ is almost $\mathbb{Z} G$-projective. Furthermore, let $H \curvearrowright Y$ be a topologically free system. If $G \curvearrowright X$ and $H \curvearrowright Y$ are continuously orbit equivalent, then they must be conjugate.

Theorem 1.4. Let $G$ be a duality group in the sense of [2, Chapter VIII, $\S 10]$ which is not infinite cyclic, and let $H$ be a finitely generated solvable group. Assume that $G \curvearrowright X$ is a topologically free system on a compact space $X$ such that $G \curvearrowright X$ is almost $\mathbb{Z} G$-projective. Furthermore, let $H \curvearrowright Y$ be a topologically free system. If $G \curvearrowright X$ and $H \curvearrowright Y$ are continuously orbit equivalent, then they must be conjugate.

Here, we say that $G \curvearrowright X$ is almost $\mathbb{Z} G$-projective if $C(X, \mathbb{Z}) \cong \mathbb{Z} \oplus P$ as $\mathbb{Z} G$-modules, where the copy of $\mathbb{Z}$ is given by the constant functions on $X$ and $P$ is a projective $\mathbb{Z} G$-module. For instance, as we will see, the Bernoulli action $G \curvearrowright X_{0}^{G}$ is almost $\mathbb{Z} G$-projective for every compact space $X_{0}$ and every torsion-free group $G$. Also, for a torsion-free group, a subshift of the full shift over a finite alphabet whose forbidden words avoid a fixed letter is almost $\mathbb{Z} G$-projective. These systems are actually even almost $\mathbb{Z} G$-free, in the sense that $P$ can be chosen to be $\mathbb{Z} G$-free. This leads to the following immediate consequence:

Corollary 1.5. Let $G \curvearrowright X$ be a topological Bernoulli action with compact base or a subshift of the full shift over a finite alphabet whose forbidden words avoid a fixed letter. Moreover, let $H \curvearrowright Y$ be a topologically free system. Assume that $G$ is a torsion-free group and that $H$ is 
a finitely generated nilpotent group which is not virtually infinite cyclic, or that $G$ is a duality group which is not infinite cyclic and that $H$ is a finitely generated solvable group. If $G \curvearrowright X$ and $H \curvearrowright Y$ are continuously orbit equivalent, then they must be conjugate.

In view of these results, an interesting and natural task is to find more examples of topological dynamical systems which are almost $\mathbb{Z} G$-projective. Apart from Bernoulli actions and subshifts, it turns out that Denjoy homeomorphisms, restricted to their unique minimal closed invariant subspaces, give rise to Cantor minimal systems which are almost $\mathbb{Z} G$-free (where $G=\mathbb{Z}$ ).

Theorem 1.3 and Theorem 1.4 are our main results. Their proofs consist of three main ingredients. Each of them is interesting in its own right.

The first ingredient establishes a link between continuous cocycle rigidity and continuous orbit equivalence rigidity. Let $G \curvearrowright X$ be a topological dynamical system, and let $H$ be a group. A continuous function $a: G \times X \rightarrow H$ is called a continuous $H$-cocycle for $G \curvearrowright X$ if $a\left(g_{1} g_{2}, x\right)=a\left(g_{1}, g_{2} \cdot x\right) a\left(g_{2}, x\right)$ for all $g_{1}, g_{2} \in G$ and $x \in X$. In particular, we can view any group homomorphism $\rho: G \rightarrow H$ as a cocycle given by $(g, x) \mapsto \rho(g)$. Continuous cocycles $a$ and $a^{\prime}$ are called cohomologous if there exists a continuous map $u: X \rightarrow H$ such that $a(g, x)=u(g . x) a^{\prime}(g, x) u(x)^{-1}$. We say that $G \curvearrowright X$ is continuous $H$-cocycle rigid if every continuous $H$-cocycle for $G \curvearrowright X$ is cohomologous to some group homomorphism $\rho: G \rightarrow H$.

In general, it is not clear how continuous cocycle rigidity is related to continuous orbit equivalence rigidity. However, we have

Theorem 1.6. Let $G$ be a torsion-free amenable group. Assume that $G \curvearrowright X$ and $H \curvearrowright Y$ are topologically free systems on compact spaces $X$ and $Y$, and suppose that $G \curvearrowright X$ and $H \curvearrowright Y$ are continuously orbit equivalent. If $G \curvearrowright X$ is continuous $H$-cocycle rigid, then $G \curvearrowright X$ and $H \curvearrowright Y$ must be conjugate.

The second ingredient establishes continuous cocycle rigidity for certain actions and certain groups.

Theorem 1.7. Let $G$ be a duality group in the sense of [2, Chapter VIII, $\S 10]$ with $c d(G) \neq 1$, let $X$ be a compact space, and suppose that $G \curvearrowright X$ is a topological dynamical system which is almost $\mathbb{Z} G$-projective. Then $G \curvearrowright X$ is continuous $H$-cocycle rigid for every solvable group $H$.

The third and final ingredient builds a bridge between continuous orbit equivalence and the notion of quasi-isometry or rather topological couplings. Inspiration comes from [7, 23, 20,

Given groups $G$ and $H$, a topological coupling $G \curvearrowright \Omega \curvearrowleft H$ for $G$ and $H$ is a locally compact Hausdorff space $\Omega$ with a left $G$-action commuting with a right $H$-action such that both actions admit compact open fundamental domains. A topological coupling $G \curvearrowright \Omega \curvearrowleft H$ is called topologically free if the corresponding $G \times H$-action is topologically free.

A continuous orbit couple for $G$ and $H$ consists of topological dynamical systems $G \curvearrowright X$, $H \curvearrowright Y$ on compact spaces $X, Y$, continuous maps $p: X \rightarrow Y, q: Y \rightarrow X, a: G \times X \rightarrow H$, $b: H \times Y \rightarrow G, g: X \rightarrow G$ and $h: Y \rightarrow H$ such that $p(g . x)=a(g, x) \cdot p(x), q(h . y)=b(h, y) \cdot q(y)$, 
$q p(x)=g(x) . x$ and $p q(y)=h(y) . y$. We call a continuous orbit couple for $G$ and $H$ topologically free if $G \curvearrowright X$ and $H \curvearrowright Y$ are topologically free. If $G \curvearrowright X$ and $H \curvearrowright Y$ are continuously orbit equivalent via $\varphi: X \cong Y$, then we obtain a continuous orbit couple for $G$ and $H$ by setting $p=\varphi$ and $q=\varphi^{-1}$.

Theorem 1.8. Let $G$ and $H$ be groups. Then there exists a one-to-one correspondence between (isomorphism classes of) topologically free continuous orbit couples for $G$ and $H$ and (isomorphism classes of) topologically free topological couplings for $G$ and $H$.

In particular, if topological dynamical systems $G \curvearrowright X, H \curvearrowright Y$ on compact spaces $X, Y$ are continuously orbit equivalent, and if one of the groups $(G$ or $H)$ is finitely generated, then $G$ and $H$ must be quasi-isometric.

In $\S 2$, we introduce the notion of continuous orbit equivalence, make some general observations and prove Theorem 1.2. Moreover, we discuss known examples for continuous orbit equivalence rigidity and construct counterexamples for which continuous orbit equivalence does not imply conjugacy in $\S 3$. In $\S$ 4, we introduce the notion of continuous cocycle rigidity, study the connection to continuous orbit equivalence rigidity, and prove Theorem 1.6. In the following section ( $(5)$, we show that Bernoulli actions, certain subshifts as well as Cantor minimal systems arising from Denjoy homeomorphisms are almost $\mathbb{Z} G$-free, study continuous cocycle rigidity using non-abelian group cohomology and prove Theorem 1.7. Thereafter, we introduce the notions of topological couplings and continuous orbit couples, establish the connection between them, and prove Theorem 1.8. Finally, in $\S$, we prove Theorem 1.3 and Theorem 1.4 .

I would like to thank David Kerr for inspiring discussions about continuous orbit equivalence.

\section{Continuous orbit equivalence, transformation groupoids and Cartan pairs}

All our groups are discrete and countable, and all our topological spaces are locally compact and Hausdorff. By a topological dynamical system, we mean an action of a group on a topological space by homeomorphisms. In this section, all our topological spaces are second countable.

Let $G \curvearrowright X$ be a topological dynamical system. The $G$-action is denoted by $G \times X \rightarrow$ $X,(g, x) \mapsto g . x$. For $x \in X$, let $G_{x}=\{g \in G: g \cdot x=x\}$ be its stabilizer group. The transformation groupoid $G \ltimes X$ attached to $G \curvearrowright X$ is given by the set $G \times X$ with multiplication $\left(g^{\prime}, x^{\prime}\right)(g, x)=\left(g^{\prime} g, x\right)$ if $x^{\prime}=g \cdot x$, inversion $(g, x)^{-1}=\left(g^{-1}, g \cdot x\right)$, range map $r(g, x)=g \cdot x$ and source map $s(g, x)=x$. Obviously, $G \ltimes X$ is étale. The reduced groupoid $\mathrm{C}^{*}$-algebra $C_{r}^{*}(G \ltimes X)$ is canonically isomorphic to $C_{0}(X) \rtimes_{r} G$. Moreover, we have a canonical embedding $C_{0}(X) \hookrightarrow C_{0}(X) \rtimes_{r} G$.

Definition 2.1. $G \curvearrowright X$ is called topologically free if for every $e \neq g \in G,\{x \in X: g . x \neq x\}$ is dense in $X$. 
From now on, for the sake of brevity, we write "topologically free system" for "topologically free topological dynamical system".

Lemma 2.2. $G \curvearrowright X$ is topologically free if and only if $\left\{x \in X: G_{x}=\{e\}\right\}$ is dense in $X$.

Proof. " $\Leftarrow "$ is clear. For " $\Rightarrow$ ", note that by topological freeness, $\{x \in X: g \cdot x \neq x\}$ is dense (and open) in $X$ for all $e \neq g \in G$. Thus, by the Baire category theorem,

$$
\left\{x \in X: G_{x}=\{e\}\right\}=\bigcap_{e \neq g \in G}\{x \in X: g \cdot x \neq x\}
$$

must be dense in $X$.

Corollary 2.3. $G \curvearrowright X$ is topologically free if and only if the transformation groupoid $G \ltimes X$ is topologically principal.

Proof. By definition (see [19]), $G \ltimes X$ is topologically principal if and only if the set of points in $X$ with trivial isotropy is dense in $X$. But this set coincides with $\left\{x \in X: G_{x}=\{e\}\right\}$. Thus Lemma 2.2 implies our corollary.

Remark 2.4. Corollary 2.3 shows that if $G \curvearrowright X$ is topologically free, then the pair $\left(C_{0}(X) \rtimes_{r}\right.$ $\left.G, C_{0}(X)\right)$ is a Cartan pair in the sense of [19, Definition 5.1].

Recall the following definition from the introduction:

Definition 2.5. Topological dynamical systems $G \curvearrowright X$ and $H \curvearrowright Y$ are continuously orbit equivalent (we write $G \curvearrowright X \sim_{\text {coe }} H \curvearrowright Y$ ) if there exists a homeomorphism $\varphi: X \stackrel{\cong}{\longrightarrow} Y$ with inverse $\psi=\varphi^{-1}: Y \stackrel{\cong}{\longrightarrow} X$ and continuous maps $a: G \times X \rightarrow H, b: H \times Y \rightarrow G$ such that

$$
\begin{aligned}
& \varphi(g . x)=a(g, x) \cdot \varphi(x) \\
& \psi(h . y)=b(h, y) \cdot \psi(y)
\end{aligned}
$$

for all $g \in G, x \in X, h \in H$ and $y \in Y$.

Remark 2.6. (1) implies $\varphi(G . x) \subseteq H . \varphi(x)$ for all $x \in X$, and (2) implies $\psi(H . y) \subseteq G . \psi(y)$ for all $y \in Y$. Thus, $\varphi(G . x)=H . \varphi(x)$ and $\psi(H . y)=G . \psi(y)$.

Remark 2.7. If $H \curvearrowright Y$ is topologically free, then $a$ is uniquely determined by (11), and by symmetry, if $G \curvearrowright X$ is topologically free, then $b$ is uniquely determined by (2). The reason is as follows: Suppose that $a^{\prime}: G \times X \rightarrow H$ is another continuous map with $\varphi(g . x)=a^{\prime}(g, x) \cdot \varphi(x)$. For arbitrary $g \in G$ and $x \in X$, there exists an open neighbourhood $U$ of $x$ such that $a$ and $a^{\prime}$ are constant on $\{g\} \times U$, with values $h$ and $h^{\prime}$ in $H$, say. Then for every $\bar{x} \in U$, $\varphi(g . \bar{x})=h \cdot \varphi(\bar{x})=h^{\prime} \cdot \varphi(\bar{x})$. Topological freeness implies $h=h^{\prime}$, in particular $a(g, x)=a^{\prime}(g, x)$.

Lemma 2.8. In Definition 2.5, if $H \curvearrowright Y$ is topologically free, then

$$
a\left(g_{1} g_{2}, x\right)=a\left(g_{1}, g_{2} . x\right) a\left(g_{2}, x\right)
$$

for all $g_{1}, g_{2} \in G$ and $x \in X$. 
Proof. Let $g_{1}, g_{2} \in G$ and $x \in X$ be arbitrary. Choose an open neighbourhood $U$ of $x \in X$ such that $a\left(g_{1} g_{2}, \bar{x}\right)=a\left(g_{1} g_{2}, x\right), a\left(g_{1}, g_{2} . \bar{x}\right)=a\left(g_{1}, g_{2} \cdot x\right)$ and $a\left(g_{2}, \bar{x}\right)=a\left(g_{2}, x\right)$ for all $\bar{x} \in U$. Then for all $\bar{x} \in U, \varphi\left(g_{1} g_{2} \cdot \bar{x}\right)=\varphi\left(g_{1} \cdot\left(g_{2} \cdot \bar{x}\right)\right)=a\left(g_{1}, g_{2} \cdot \bar{x}\right) \cdot \varphi\left(g_{2} \cdot \bar{x}\right)=a\left(g_{1}, g_{2} \cdot \bar{x}\right) a\left(g_{2}, \bar{x}\right) \cdot \varphi(\bar{x})=$ $a\left(g_{1}, g_{2} \cdot x\right) a\left(g_{2}, x\right) \cdot \varphi(\bar{x})$, but also $\varphi\left(g_{1} g_{2} \cdot \bar{x}\right)=a\left(g_{1} g_{2}, \bar{x}\right) \cdot \varphi(\bar{x})=a\left(g_{1} g_{2}, x\right) \cdot \varphi(\bar{x})$. By topological freeness, $a\left(g_{1} g_{2}, x\right)=a\left(g_{1}, g_{2} \cdot x\right) a\left(g_{2}, x\right)$.

Lemma 2.9. In the situation of Definition [2.5, let $Y_{f}=\left\{y \in Y: H_{y}=\{e\}\right\}$. For every $x \in$ $\psi\left(Y_{f}\right), a_{x}: G \rightarrow H, g \mapsto a(g, x)$ is bijective.

Proof. Since $\varphi(x) \in Y_{f}, a_{x}$ is injective. To prove surjectivity, take $h \in H$. Since by Remark 2.6, $\varphi(G . x)=H \cdot \varphi(x)$, there exists $g \in G$ with $h \cdot \varphi(x)=\varphi(g . x)=a(g, x) \cdot \varphi(x)$. As $\varphi(x) \in Y_{f}$, we conclude that $h=a(g, x)=a_{x}(g)$.

Lemma 2.10. In the situation of Definition [2.5, assume that $G \curvearrowright X$ and $H \curvearrowright Y$ are topologically free. Then

$$
b(a(g, x), \varphi(x))=g \text { for all } g \in G, x \in X,
$$

and $b$ is uniquely determined by (3).

Proof. Let $h:=a(g, x)$. Then $\varphi(g . x)=h . \varphi(x)$, so $g . x=\psi(h . \varphi(x))=b(h, \varphi(x)) . x$. Since this equation holds in an open neighbourhood of $x$, topological freeness implies $b(a(g, x), \varphi(x))=g$. Moreover, note that for all $x \in \psi\left(Y_{f}\right), a_{x}(G)=H$ by Lemma 2.9. Hence (3) determines $b$ on $H \times Y_{f}$. But since $Y_{f}$ is dense in $Y$ by topological freeness, and because $b$ is continuous, (3) determines $b$ on $H \times Y$.

Corollary 2.11. In the situation of Definition [2.5, assume that $G \curvearrowright X$ and $H \curvearrowright Y$ are topologically free. Let $X_{f}=\left\{x \in X: G_{x}=\{e\}\right\}$ and $Y_{f}=\left\{y \in Y: H_{y}=\{e\}\right\}$. Then $\varphi\left(X_{f}\right)=$ $Y_{f}$. In particular, for every $x \in X$ with $G_{x}=\{e\}, a_{x}: G \rightarrow H, g \mapsto a(g, x)$ is bijective.

Proof. By symmetry, we just have to show $\varphi\left(X_{f}\right) \subseteq Y_{f}$. Take $x \in X_{f}$, and let $y=\varphi(x)$. Suppose that $h \in H$ satisfies $h . y=y$. Then $x=\psi(y)=\psi(h . y)=b(h, y) \cdot \psi(y)=b(h, y) . x$, and therefore $b(h, y)=e$ since $x \in X_{f}$. But by the analogue of (3) with reversed roles for $a$ and $b$, we get $e=a(e, x)=a(b(h, y), x)=h$. Hence $y \in Y_{f}$.

We are now ready for the proof of Theorem 1.2 .

Theorem (Theorem 1.2). Let $G \curvearrowright X$ and $H \curvearrowright Y$ be topologically free systems. The following are equivalent:

(i) $G \curvearrowright X \sim$ coe $H \curvearrowright Y$;

(ii) $G \ltimes X \cong H \ltimes Y$ (as topological groupoids);

(iii) there is a $C^{*}$-isomorphism $\Phi: C_{0}(X) \rtimes_{r} G \stackrel{\cong}{\longrightarrow} C_{0}(Y) \rtimes_{r} H$ with $\Phi\left(C_{0}(X)\right)=C_{0}(Y)$.

Proof. (i) $\Rightarrow$ (ii): Assume that $G \curvearrowright X \sim_{\text {coe }} H \curvearrowright Y$, and let $\varphi, \psi, a$ and $b$ be as in Definition 2.5. Then $G \ltimes X \rightarrow H \ltimes Y,(g, x) \mapsto(a(g, x), \varphi(x))$ and $H \ltimes Y \rightarrow G \ltimes X,(h, y) \mapsto$ 
$(b(h, y), \psi(y))$ are certainly continuous groupoid morphisms, and they are inverse to each other due to (3) and the analogue of (3) with reversed roles for $a$ and $b$.

(ii) $\Rightarrow$ (i): Let $\chi: G \ltimes X \stackrel{\cong}{\longrightarrow} H \ltimes Y$ be an isomorphism of topological groupoids. Set $\varphi=\left.\chi\right|_{X}: X \stackrel{\cong}{\longrightarrow} Y$ and let $a$ be the composition $G \ltimes X \stackrel{\chi}{\longrightarrow} H \ltimes Y \rightarrow H$, where the second map is $H \ltimes Y \rightarrow H,(h, y) \mapsto h$. Then $a$ is obviously continuous, and $\varphi(g . x)=$ $\chi(r(g, x))=r(\chi(g, x))=r(a(g, x), \varphi(x))=a(g, x) \cdot \varphi(x)$. Similarly, for $\psi=\varphi^{-1}$, if we let $b$ be the composition $H \ltimes Y \stackrel{\chi^{-1}}{\longrightarrow} G \ltimes X \rightarrow G$, where the second map is $G \ltimes X \rightarrow G,(g, x) \mapsto g$, then $\psi(h . y)=b(h, y) . \psi(y)$.

(ii) $\Leftrightarrow$ (iii) is [19, Proposition 4.13], where we have to use Corollary 2.3,

\section{Continuous orbit equivalence Rigidity: Examples And COUnterexamples}

Let us compare continuous orbit equivalence with conjugacy.

Definition 3.1. Topological dynamical systems $G \curvearrowright X$ and $H \curvearrowright Y$ are conjugate (we write $G \curvearrowright X \sim_{\text {conj }} H \curvearrowright Y$ ) if there is a homeomorphism $\varphi: X \stackrel{\cong}{\longrightarrow} Y$ and a group isomorphism $\rho: G \stackrel{\cong}{\longrightarrow} H$ such that for every $g \in G$ and $x \in X, \varphi(g . x)=\rho(g) \cdot \varphi(x)$.

Obviously, $G \curvearrowright X \sim_{\text {conj }} H \curvearrowright Y$ implies $G \curvearrowright X \sim_{\text {coe }} H \curvearrowright Y$. Are there classes of dynamical systems where we can reverse this implication, i.e., where continuous orbit equivalence implies conjugacy?

Here is a first class of examples, for which continuous orbit equivalence rigidity holds because of a trivial reason: Suppose that $G \curvearrowright X$ is a topologically free system on a connected space $X$. If $G \curvearrowright X \sim_{\text {coe }} H \curvearrowright Y$ for some topologically free system $H \curvearrowright Y$, then $G \curvearrowright X \sim_{\text {conj }} H \curvearrowright Y$. The reason is that the function $a$ in Definition 2.5 is continuous, hence for every $g \in G,\left.a\right|_{\{g\} \times X}$ is constant because $X$ is connected and $H$ is discrete. Hence $a(g, x)=\rho(g)$ for some map $\rho: G \rightarrow H$, and $\rho$ has to be a homomorphism (by Lemma 2.8) and bijective (by Lemmma 2.9).

This observation means that if we focus on discrete groups, it is natural to restrict our discussion to topological dynamical systems on totally disconnected spaces.

Here is a first result in continuous orbit equivalence rigidity:

Theorem 3.2 ([1, Theorem 3.2]). Let $\mathbb{Z} \curvearrowright X$ and $\mathbb{Z} \curvearrowright Y$ be topologically free systems on compact spaces $X$ and $Y$. Assume that $\mathbb{Z} \curvearrowright X$ is topologically transitive.

If $\mathbb{Z} \curvearrowright X \sim_{\text {coe }} \mathbb{Z} \curvearrowright Y$, then $\mathbb{Z} \curvearrowright X \sim_{\text {conj }} \mathbb{Z} \curvearrowright Y$.

In this theorem, while the groups are fixed, the assumptions on the actions are very mild. Therefore, an immediate question is whether there are counterexamples to continuous orbit 
equivalence rigidity at all, i.e., examples of topological dynamical systems which are continuously orbit equivalent but not conjugate.

3.1. Products of odometer transformations. Let $M=\prod_{p} p^{v_{p}}$ be a supernatural number. Here, the product is taken over all primes, $v_{p} \in\{0,1,2, \ldots\} \cup\{\infty\}$, and $\sum_{p} v_{p}=\infty$. The odometer action $\mathbb{Z} \curvearrowright \mathbb{Z} / M$ corresponding to $M$ is constructed as follows:

Choose a sequence $\left(m_{k}\right)_{k}$ of natural numbers such that, for all primes $p, v_{p}\left(m_{k}\right) \nearrow v_{p}$ for $k \rightarrow \infty$. Then set $\mathbb{Z} / M=\lim _{k} \mathbb{Z} / m_{k}$. The canonical projections $\mathbb{Z} \rightarrow \mathbb{Z} / m_{k}$ induce a group embedding $\mathbb{Z} \hookrightarrow \mathbb{Z} / M$, and this in turn yields an action $\mathbb{Z} \curvearrowright \mathbb{Z} / M$ which we call the odometer transformation for $M$.

Theorem 3.3. For supernatural numbers $M_{1}, \ldots, M_{r}$ and $N_{1}, \ldots, N_{s}$, the following are equivalent:

(i) $\mathbb{Z}^{r} \curvearrowright \prod_{i=1}^{r} \mathbb{Z} / M_{i} \sim_{\text {coe }} \mathbb{Z}^{s} \curvearrowright \prod_{j=1}^{s} \mathbb{Z} / N_{j}$;

(ii) $C_{0}\left(\prod_{i=1}^{r} \mathbb{Z} / M_{i}\right) \rtimes \mathbb{Z}^{r} \cong C_{0}\left(\prod_{j=1}^{s} \mathbb{Z} / N_{j}\right) \rtimes \mathbb{Z}^{s}$;

(iii) $\left(K_{*}\left(C_{0}\left(\prod_{i=1}^{r} \mathbb{Z} / M_{i}\right) \rtimes \mathbb{Z}^{r}\right),[1]_{0}\right) \cong\left(K_{*}\left(C_{0}\left(\prod_{j=1}^{s} \mathbb{Z} / N_{j}\right) \rtimes \mathbb{Z}^{s}\right),[1]_{0}\right)$;

(iv) $r=s$, there exists $\sigma \in S_{r}$, natural numbers $m_{1}, \ldots, m_{r}$ and $n_{1}, \ldots, n_{r}$ such that for all $1 \leq i \leq r, m_{i} M_{i}=n_{\sigma(i)} N_{\sigma(i)}$, and $\prod_{i=1}^{r} M_{i}=\prod_{j=1}^{r} N_{j}$

Proof. (i) $\Rightarrow$ (ii) follows from Theorem 1.2 as our systems are free.

(ii) $\Rightarrow$ (iii) is clear.

(iii) $\Rightarrow$ (iv): $K_{*}$ stands for $K_{0} \oplus K_{1}$. Clearly, $\left(K_{0}(C(\mathbb{Z} / M) \rtimes \mathbb{Z}),[1]_{0}\right) \cong\left(\mathbb{Z}\left[M^{-1}\right], 1\right)$ and $K_{1}(C(\mathbb{Z} / M) \rtimes \mathbb{Z}) \cong \mathbb{Z}$. Here $\mathbb{Z}\left[M^{-1}\right]=\left\{\frac{x}{m} \in \mathbb{Q}: m \mid M\right\}$. So $K_{*}\left(C\left(\prod_{i=1}^{r} \mathbb{Z} / M_{i}\right) \rtimes \mathbb{Z}^{r}\right) \cong$ $\bigoplus_{I \subseteq\{1, \ldots, r\}} \mathbb{Z}\left[\left(\prod_{i \in I} M_{i}\right)^{-1}\right]$ and $[1]_{0}$ corresponds to $1 \in \mathbb{Z}\left[\left(\prod_{i=1}^{r} M_{i}\right)^{-1}\right](I=\{1, \ldots, r\})$.

Therefore, $\mathbb{Q}^{2^{r}} \cong K_{*}\left(C\left(\prod_{i=1}^{r} \mathbb{Z} / M_{i}\right) \rtimes \mathbb{Z}^{r}\right) \otimes \mathbb{Q} \cong K_{*}\left(C\left(\prod_{j=1}^{s} \mathbb{Z} / N_{j}\right) \rtimes \mathbb{Z}^{s}\right) \otimes \mathbb{Q} \cong \mathbb{Q}^{2^{s}}$, and this implies $r=s$. Moreover, as a $K_{*}$-isomorphism preserves $[1]_{0}$, it restricts to an isomorphism $\mathbb{Z}\left[\left(\prod_{i=1}^{r} M_{i}\right)^{-1}\right] \cong \mathbb{Z}\left[\left(\prod_{j=1}^{r} N_{j}\right)^{-1}\right]$ sending 1 to 1 . This implies $\prod_{i=1}^{r} M_{i}=\prod_{j=1}^{r} N_{j}$.

Given supernatural numbers $M$ and $N$, we define $M \lesssim N$ if there exists $n \in \mathbb{N}$ with $M$ $n N\left(v_{p}(M) \leq v_{p}(n N)\right)$. We define $M \sim N$ if $M \lesssim N$ and $N \lesssim M$. It is immediate that there exists a non-zero homomorphism $\mathbb{Z}\left[M^{-1}\right] \rightarrow \mathbb{Z}\left[N^{-1}\right]$ if and only if $M \lesssim N$. Set $\mathcal{M}=\left\{M_{i}: 1 \leq i \leq r\right\}, \wedge \mathcal{M}=\left\{\prod_{i \in I} M_{i}: I \subseteq\{1, \ldots, r\}\right\}$ and $\mathcal{N}=\left\{N_{j}: 1 \leq j \leq r\right\}$, $\bigwedge \mathcal{N}=\left\{\prod_{j \in J} N_{j}: J \subseteq\{1, \ldots, r\}\right\}$. Using the assumption that $\bigoplus_{I \subseteq\{1, \ldots, r\}} \mathbb{Z}\left[\left(\prod_{i \in I} M_{i}\right)^{-1}\right] \cong$ $\bigoplus_{J \subseteq\{1, \ldots, r\}} \mathbb{Z}\left[\left(\prod_{j \in J} N_{j}\right)^{-1}\right]$, a straightforward inductive argument shows that for every equivalence class $\mathcal{S}$ of supernatural numbers with respect to $\sim, \mathcal{S} \cap \wedge \mathcal{M}|=| \mathcal{S} \cap \wedge \mathcal{N} \mid$, and then also $|\mathcal{S} \cap \mathcal{M}|=|\mathcal{S} \cap \mathcal{N}|$. 
(iv) $\Rightarrow$ (i): We need the following observation: Let $l$ be a natural number and $\lambda_{l}: \mathbb{Z} / l \curvearrowright \mathbb{Z} / l$ the canonical action. Let $L$ be a supernatural number, $X=\mathbb{Z} / l L, \tilde{X}=l \cdot(\mathbb{Z} / l L), \alpha_{l L}: \mathbb{Z} \curvearrowright X$ the odometer transformation for $l L$, and $\tilde{\alpha}=\left.\alpha\right|_{L \mathbb{Z}}: l \mathbb{Z} \curvearrowright \tilde{X}$. We claim that

$$
\alpha_{l L} \sim_{\text {coe }} \lambda_{l} \otimes \tilde{\alpha}_{l L} \sim_{\text {conj }} \lambda_{l} \otimes \alpha_{L}
$$

$\bigotimes$ stand for the product action. Let us prove (44). Define $\varphi: X=\bigsqcup_{k=0}^{l-1} k+\tilde{X} \rightarrow \mathbb{Z} / l \times \tilde{X}, k+x \mapsto$ $([k], x)$. It is easy to see that the inverse of $\varphi$ is given by $\psi: \mathbb{Z} / l \times \tilde{X} \rightarrow X,([k], x) \mapsto k+x$ for $0 \leq k \leq l-1$. Moreover, define $a: \mathbb{Z} \times X=\bigsqcup_{j=0}^{l-1}(j+l \mathbb{Z}) \times \bigsqcup_{k=0}^{l-1}(k+\tilde{X}) \rightarrow \mathbb{Z} / l \times l \mathbb{Z}$ by setting $a(j+h, k+x)=([j], h)$ if $j+k \leq l-1$ and $a(j+h, k+x)=([j], k+l)$ if $l<j+k$. Also, define $b:(\mathbb{Z} / l \times l \mathbb{Z}) \times(\mathbb{Z} / l \times \tilde{X}) \rightarrow \mathbb{Z}$ by setting $b(([j], h),([k], x))=j+h$ if $j+k \leq l-1$ and $b(([j], h),([k], x))=j+h-l$ if $l \leq j+k$, where $0 \leq j, k \leq l-1$. Then it is easy to check that $\varphi$, $a, \psi$ and $b$ satisfy (11) and (2), so that $\alpha_{l L} \sim_{\text {coe }} \lambda_{l} \otimes \tilde{\alpha}_{l L}$. Furthermore, $\lambda_{l} \otimes \tilde{\alpha}_{l L} \sim_{\text {conj }} \lambda_{l} \otimes \alpha_{L}$ is easy to see. This proves (4).

Now we can complete the proof for (iv) $\Rightarrow$ (i). Without loss of generality we may assume that $\sigma=$ id, i.e., $m_{i} M_{i}=n_{i} N_{i}$ for all $1 \leq i \leq r$. Without loss of generality, we may further assume $\operatorname{gcd}\left(m_{i}, n_{i}\right)=1$. Then we can write $M_{i}=n_{i} L_{i}$ and $N_{i}=m_{i} L_{i}$ for some supernatural number $L_{i}$. Set $L=\prod_{i=1}^{r} L_{i}$, and choose natural numbers $m$ and $n$ with $\operatorname{gcd}(m, L)=1=\operatorname{gcd}(n, L)$ such that $\prod_{i=1}^{r} M_{i}=\left(\prod_{i=1}^{r} n_{i}\right)\left(\prod_{i=1}^{r} L_{i}\right)=n L$ and $\prod_{j=1}^{r} N_{j}=\left(\prod_{j=1}^{r} m_{j}\right)\left(\prod_{j=1}^{r} L_{j}\right)=m L$. $\prod_{i=1}^{r} M_{i}=\prod_{j=1}^{r} N_{j}$ implies that $m=n$. Therefore, we get

$$
\begin{aligned}
\bigotimes_{i=1}^{r} \alpha_{M_{i}} & =\bigotimes_{i=1}^{r} \alpha_{n_{i} L_{i}} \stackrel{(4)}{\sim}_{\text {coe }} \bigotimes_{i=1}^{r}\left(\lambda_{n_{i}} \otimes \alpha_{L_{i}}\right) \stackrel{(4)}{\sim}_{\text {coe }} \alpha_{n L_{1}} \otimes \alpha_{L_{2}} \otimes \ldots \otimes \alpha_{L_{r}} \\
& =\alpha_{m L_{1}} \otimes \alpha_{L_{2}} \otimes \ldots \otimes \alpha_{L_{r}} \stackrel{(4)}{\sim}_{\text {coe }} \otimes_{j=1}^{r} \alpha_{N_{j}} .
\end{aligned}
$$

In contrast, for conjugacy, we get

Theorem 3.4. Let $I$ and $J$ be finite sets. For supernatural numbers $\left\{M_{i}\right\}_{i \in I}$ and $\left\{N_{j}\right\}_{j \in J}$, $\mathbb{Z}^{I} \curvearrowright \prod_{i \in I} \mathbb{Z} / M_{i} \sim_{\text {conj }} \mathbb{Z}^{J} \curvearrowright \prod_{j \in J} \mathbb{Z} / N_{j}$ if and only if there exists a finite set $K$, supernatural numbers $\left\{L_{k}\right\}_{k \in K}$ such that

- $I=\bigsqcup_{k \in K} I_{k}, J=\bigsqcup_{k \in K} J_{k}$,

- $\left|I_{k}\right|=\left|J_{k}\right|$ for all $k \in K$,

- for every $k \in K$, every $i \in I_{k}$ and $j \in J_{k}$, we can write $M_{i}=m_{i} L_{k}$ and $N_{j}=n_{j} L_{k}$ for some (uniquely determined) $m_{i}, n_{j} \in \mathbb{N}$ with $\operatorname{gcd}\left(m_{i}, L_{k}\right)=1=\operatorname{gcd}\left(n_{j}, L_{k}\right)$, and we have $\prod_{i \in I_{k}} \mathbb{Z} / m_{i} \cong \prod_{j \in J_{k}} \mathbb{Z} / n_{j}$.

Proof. " $\Rightarrow "$ : Assume that $\rho: \mathbb{Z}^{I} \cong \mathbb{Z}^{J}$ is a group isomorphism and $\varphi: \prod_{i \in I} \mathbb{Z} / M_{i} \cong \prod_{j \in J} \mathbb{Z} / N_{j}$ such that $\varphi(g \cdot x)=\rho(g) \cdot \varphi(x)$. $\mathbb{Z}^{I} \cong \mathbb{Z}^{J}$ implies that $|I|=|J|$. Set $r=|I|=|J|$. Moreover, we may assume $\varphi(0)=0$ (otherwise go over to $\varphi-\varphi(0)$ ). Let $\rho$ be multiplication with $S \in G L_{r}(\mathbb{Z})$. 
It is straightforward to check that if $S_{j, i} \neq 0$, then $N_{j} \lesssim M_{i}$. So there exist a finite set $K$ and decompositions $I=\bigsqcup_{k \in K} I_{k}, J=\bigsqcup_{k \in K} J_{k}$ with $\left|I_{k}\right|=\left|J_{k}\right|$ for all $k \in K$ such that for every $(i, j) \in I_{k} \times J_{k}, M_{i} \sim N_{j}$.

Fix $k \in K$. Find a supernatural number $L_{k}$ such that for every $i \in I_{k}, j \in J_{k}$, there are $m_{i} \in \mathbb{N}$, $n_{j} \in \mathbb{N}$ with $\operatorname{gcd}\left(m_{i}, L_{k}\right)=1=\operatorname{gcd}\left(n_{j}, L_{k}\right)$ such that $M_{i}=m_{i} L_{k}$ and $N_{j}=n_{j} L_{k}$. Then $\varphi$ restricts to an isomorphism of topological abelian groups

$$
\varphi_{k}:\left(\prod_{i \in I_{k}} \mathbb{Z} / m_{i}\right) \times\left(\prod_{i \in I_{k}} \mathbb{Z} / L_{k}\right) \cong \prod_{i \in I_{k}} \mathbb{Z} / M_{i} \cong \prod_{j \in J_{k}} \mathbb{Z} / N_{j} \cong\left(\prod_{j \in J_{k}} \mathbb{Z} / n_{j}\right) \times\left(\prod_{j \in J_{k}} \mathbb{Z} / L_{k}\right) .
$$

Let $l \in \mathbb{N}$ satisfy $\prod_{j \in J_{k}} n_{j} \mid l$ and $\operatorname{gcd}(l, L)=1$. Certainly, $\varphi_{k}(w, 0)$ is of the form $(z, 0)$ as for all $i \in I_{k}$, there exists no non-zero homomorphism $\mathbb{Z} / m_{i} \rightarrow \mathbb{Z} / L_{k}$. Also, $\varphi_{k}(0, y)$ is of the form $\varphi_{k}(0, l \cdot \tilde{y})=l \cdot \varphi_{k}(0, \tilde{y})$, hence of the form $(0, x)$ as for all $j \in J_{k}, l \equiv 0$ in $\mathbb{Z} / n_{j}$. Hence $\varphi_{k}=\phi_{t} \times \phi_{L}$ for some group isomorphisms $\phi_{t}: \prod_{i \in I_{k}} \mathbb{Z} / m_{i} \cong \prod_{j \in J_{k}} \mathbb{Z} / n_{j}$ and $\phi_{L}: \prod_{i \in I_{k}} \mathbb{Z} / L_{k} \stackrel{\cong}{\longrightarrow} \prod_{j \in J_{k}} \mathbb{Z} / L_{k}$.

" $\Leftarrow "$ : Without loss of generality we may assume $|K|=1$. Let $K=\{k\}, I=I_{k}, J=J_{k}$, $|I|=|J|=r$. We may assume that $I=J=\{1, \ldots, r\}$. Let $L=L_{k}$ be a supernatural number such that for every $1 \leq i, j \leq r, M_{i}=m_{i} L$ and $N_{j}=n_{j} L$ for some (unique) $m_{i}, n_{j} \in \mathbb{N}$ with $\operatorname{gcd}\left(m_{i}, L\right)=1=\operatorname{gcd}\left(n_{j}, L\right)$, and such that $\prod_{i=1}^{r} \mathbb{Z} / m_{i} \cong \prod_{j=1}^{r} \mathbb{Z} / n_{j}$. By the theory of elementary divisors, there are $S, T \in G L_{r}(\mathbb{Z})$ such that $S\left(\begin{array}{ccc}m_{1} & & 0 \\ & \ddots & \\ 0 & & m_{r}\end{array}\right) T=\left(\begin{array}{ccc}n_{1} & & 0 \\ & \ddots & \\ 0 & & n_{r}\end{array}\right)$. Thus $S\left(\left(\begin{array}{ccc}m_{1} & & 0 \\ & \ddots & \\ 0 & & m_{r}\end{array}\right) \mathbb{Z}^{r}\right)=\left(\begin{array}{ccc}n_{1} & & 0 \\ & \ddots & \\ 0 & & n_{r}\end{array}\right) \mathbb{Z}^{r}$. So the same matrix $S$ induces two group isomorphisms $\rho: \mathbb{Z}^{r} \rightarrow \mathbb{Z}^{r}, g \mapsto S g$ and $\phi_{t}: \prod_{i=1}^{r} \mathbb{Z} / m_{i} \cong \mathbb{Z}^{r} /\left(\begin{array}{ccc}m_{1} & & 0 \\ & \ddots & \\ 0 & & m_{r}\end{array}\right) \mathbb{Z}^{r} \cong \mathbb{Z}^{r} /\left(\begin{array}{ccc}n_{1} & & 0 \\ & \ddots & \\ 0 & & n_{r}\end{array}\right) \mathbb{Z}^{r} \cong$ $\prod_{i=j}^{r} \mathbb{Z} / n_{j}$ as well as an isomorphism of topological groups $\phi_{L}:(\mathbb{Z} / L)^{r} \cong(\mathbb{Z} / L)^{r}$. Let $\varphi$ be the isomorphism

$$
\begin{aligned}
& \prod_{i=1}^{r} \mathbb{Z} / M_{i} \cong \prod_{i=1}^{r}\left(\mathbb{Z} / m_{i} \times \mathbb{Z} / L\right) \cong\left(\prod_{i=1}^{r} \mathbb{Z} / m_{i}\right) \times(\mathbb{Z} / L)^{r} \\
\stackrel{\phi_{t} \times \phi_{L}}{\longrightarrow} & \left(\prod_{j=1}^{r} \mathbb{Z} / n_{j}\right) \times(\mathbb{Z} / L)^{r} \cong \prod_{j=1}^{r}\left(\mathbb{Z} / n_{j} \times \mathbb{Z} / L\right) \cong \prod_{j=1}^{r} \mathbb{Z} / N_{j} .
\end{aligned}
$$

Then $\left.\varphi\right|_{\mathbb{Z}^{r}}=\rho$, and so $\varphi(g . x)=\rho(g) . \varphi(x)$ for all $g \in \mathbb{Z}^{r}$ and $x \in \prod_{i=1}^{r} \mathbb{Z} / M_{i}$. This means that $\mathbb{Z}^{I} \curvearrowright \prod_{i \in I} \mathbb{Z} / M_{i} \sim_{\text {conj }} \mathbb{Z}^{J} \curvearrowright \prod_{j \in J} \mathbb{Z} / N_{j}$.

Comparing Theorem 3.3 and Theorem 3.4, we can easily construct products of odometers which are continuously orbit equivalent but not conjugate.

Example 3.5. Let $r \geq 2$. Let $p$ and $q$ be primes, $p \neq q$, and let $n \in \mathbb{N}$ with $n>1$ and $\operatorname{gcd}(p, n)=1=\operatorname{gcd}(q, n)$. If we set $M_{1}=n \cdot p^{\infty}, M_{2}=q^{\infty}, M_{3}=\ldots=M_{s}=p^{\infty}$ and 
$N_{1}=p^{\infty}, N_{2}=n \cdot q^{\infty}, N_{3}=\ldots=N_{s}=p^{\infty}$, then $\mathbb{Z}^{r} \curvearrowright \prod_{i=1}^{r} \mathbb{Z} / M_{i} \sim_{\text {coe }} \mathbb{Z}^{r} \curvearrowright \prod_{j=1}^{r} \mathbb{Z} / N_{j}$ but $\mathbb{Z}^{r} \curvearrowright \prod_{i=1}^{r} \mathbb{Z} / M_{i} \nsim_{\text {conj }} \mathbb{Z}^{r} \curvearrowright \prod_{j=1}^{r} \mathbb{Z} / N_{j}$.

3.2. Actions of non-abelian free groups. Let us construct actions of the free group $\mathbb{F}_{r}$ $(r \geq 2)$ on the Cantor set, which are continuously orbit equivalent but not conjugate. Let $a_{1}, \ldots, a_{r}$ be generators of $\mathbb{F}_{r}$. Let $\beta: \mathbb{F}_{r} \curvearrowright \partial \mathbb{F}_{r}$ be the $\mathbb{F}_{r}$-action on the Gromov boundary of $\mathbb{F}_{r}$, and set $\beta_{i}:=\beta_{a_{i}}$. For a supernatural number $M$, let $\alpha_{M}: \mathbb{Z} \curvearrowright \mathbb{Z} / M$ be the corresponding odometer transformation. For supernatural numbers $M_{1}, M_{2}, N_{1}$ and $N_{2}$, define actions $\gamma$ : $\mathbb{F}_{r} \curvearrowright \partial \mathbb{F}_{r} \times\left(\mathbb{Z} / M_{1}\right) \times\left(\mathbb{Z} / M_{2}\right)$ and $\delta: \mathbb{F}_{r} \curvearrowright \partial \mathbb{F}_{r} \times\left(\mathbb{Z} / N_{1}\right) \times\left(\mathbb{Z} / N_{2}\right)$ by setting $\gamma_{1}:=\beta_{1} \times \alpha_{M_{1}} \times$ id, $\gamma_{2}:=\beta_{2} \times$ id $\times \alpha_{M_{2}}, \gamma_{i}:=\beta_{i} \times$ id $\times$ id for all $i \geq 3, \gamma_{a_{i}}=\gamma_{i}$ for all $1 \leq i \leq r$, and similarly $\delta_{1}:=\beta_{1} \times \alpha_{N_{1}} \times$ id, $\delta_{2}:=\beta_{2} \times$ id $\times \alpha_{N_{2}}, \delta_{i}:=\beta_{i} \times$ id $\times$ id for all $i \geq 3, \delta_{a_{i}}=\delta_{i}$ for all $1 \leq i \leq r$.

Theorem 3.6. Let $p$ and $q$ be primes, $p \neq q$, and let $n \in \mathbb{N}$ with $n>1$ and $\operatorname{gcd}(p, n)=1=$ $\operatorname{gcd}(q, n)$. If we set $M_{1}=n \cdot p^{\infty}, M_{2}=q^{\infty}$ and $N_{1}=p^{\infty}, N_{2}=n \cdot q^{\infty}$, then $\gamma \sim_{\text {coe }} \delta$ but $\gamma \nsim_{\text {conj }} \delta$.

For the proof, we need some preparation. Let $X$ be a totally disconnected compact space. In our application, $X$ will be the Cantor space. Let $C^{\infty}(X, \mathbb{C})=C(X, \mathbb{Z}) \otimes \mathbb{C}$. Obviously, we have an isomorphism $C^{\infty}(X, \mathbb{C}) \stackrel{\cong}{\longrightarrow}\{X \stackrel{f}{\longrightarrow} \mathbb{C}$ continuous: $f(X) \subseteq \mathbb{C}$ finite $\}, f \otimes z \mapsto f \cdot z$. Here $(f$. $z)(x)=f(x) z$. In the following, we view elements in $C^{\infty}(X, \mathbb{C})$ as $\mathbb{C}$-valued continuous functions on $X$ via this explicit isomorphism. Let $\phi: X \rightarrow X$ be a homeomorphism, and denote the induced automorphism of $C(X)$ by $\phi$ again. Obviously, $\phi\left(C^{\infty}(X, \mathbb{C})\right) \subseteq C^{\infty}(X, \mathbb{C})$. We define $E(\phi):=\left\{z \in \mathbb{T}: \phi(f)=z f\right.$ for some $\left.0 \neq f \in C^{\infty}(X, \mathbb{C})\right\}$. Now let $g_{1}, \ldots, g_{r}$ be generators of $\mathbb{F}_{r}$, and let $g=g_{1}$. Let $Y$ be a totally disconnected compact space and let $\alpha: Y \cong Y$ be a homeomorphism.

Proposition 3.7. For $\phi=\beta_{g} \times \alpha$, we have $E_{\phi}=E_{\alpha}$.

Proof. We think of elements in $\partial \mathbb{F}_{r}$ as infinite reduced words in $g^{ \pm 1}=g_{1}^{ \pm 1}, g_{2}^{ \pm 1}, \ldots, g_{r}^{ \pm 1}$. Let $W$ be the set of finite reduced words in $g_{1}^{ \pm 1}, g_{2}^{ \pm 1}, \ldots, g_{r}^{ \pm 1}$ which do not end on $g_{r}^{-1}$ nor on $g_{r}^{2}$. For $w \in W$, let $C_{w}$ be the subspace of $\partial \mathbb{F}_{r}$ consisting of those infinite reduced words which start with $w$. Note that the empty word $\emptyset$ lies in $W$, and that $C_{\emptyset}=\partial \mathbb{F}_{r}$. Clearly, $\left\{1_{C_{w}}: w \in W\right\}$ is a $\mathbb{Z}$-basis for $C\left(\partial \mathbb{F}_{r}, \mathbb{Z}\right)$. Take a family $\mathcal{C}$ of compact open subsets of $Y$ such that $\left\{1_{C}: C \in \mathcal{C}\right\}$ is a $\mathbb{Z}$-basis for $C(Y, \mathbb{Z})$. Then $\left\{1_{C_{w}} \otimes 1_{C}: w \in W, C \in \mathcal{C}\right\}$ is a $\mathbb{Z}$-basis for $C\left(\partial \mathbb{F}_{r} \times Y, \mathbb{Z}\right) \cong C\left(\partial \mathbb{F}_{r}, \mathbb{Z}\right) \otimes C(Y, \mathbb{Z})$.

Let $z \in E_{\phi}$, and let $f=\sum_{i} 1_{C_{w_{i}}} \otimes 1_{C_{i}} \otimes \lambda_{i}\left(\lambda_{i} \in \mathbb{C} \backslash\{0\}\right)$ be a non-zero element in $C\left(\partial \mathbb{F}_{r} \times Y, \mathbb{C}\right) \cong$ $C\left(\partial \mathbb{F}_{r}, \mathbb{Z}\right) \otimes C(Y, \mathbb{Z}) \otimes \mathbb{C}$ with $\phi(f)=z f$. Then $\sum_{i} 1_{C_{w_{i}}} \otimes\left(1_{C_{i}} \otimes z \lambda_{i}\right)=z f=\phi(f)=$ $\sum_{i} 1_{\beta_{g}\left(C_{w_{i}}\right)} \otimes\left(1_{\alpha\left(C_{i}\right)} \otimes \lambda_{i}\right)=\sum_{j} 1_{C_{w_{j}}} \otimes f_{j}$ for some $0 \neq f_{j} \in C(Y, \mathbb{Z}) \otimes \mathbb{C}$, where $\left\{w_{j}\right\}_{j}$ are chosen so that $1_{\beta_{g}\left(C_{w_{i}}\right)} \in \mathbb{Z}$-span $\left(\left\{w_{j}\right\}_{j}\right)$ for all $i$. It follows that $\left\{w_{i}\right\}_{i}=\left\{w_{j}\right\}_{j} \supseteq\left\{g w_{i}: w_{i} \neq g^{-1}\right\} \cup\{g\}$ if $g^{-1} \in\left\{w_{i}\right\}_{i}$ and $\left\{w_{i}\right\}_{i}=\left\{w_{j}\right\}_{j} \supseteq\left\{g w_{i}: w_{i} \neq g^{-1}\right\}$ if $g^{-1} \notin\left\{w_{i}\right\}_{i}$. Here we use that $\beta_{g}\left(C_{w_{i}}\right)=C_{g w_{i}} \neq C_{w_{i}}$ if $w_{i} \neq g^{-1}$ and $\beta_{g}\left(C_{g^{-1}}\right)=\partial \mathbb{F}_{r} \backslash C_{g}$. 
We claim that it already follows that $\left\{w_{i}\right\}_{i}=\left\{\partial \mathbb{F}_{r}\right\}$ : If there is $w \in\left\{w_{i}\right\}_{i}$ not starting with $g^{-1}$, then $g^{n} w \in\left\{w_{i}\right\}$ for all $m \in \mathbb{N}$ which is impossible since $\left\{w_{i}\right\}_{i}$ is finite. If there is $w \in\left\{w_{i}\right\}_{i}$ of the form $g^{-m} v$ where $v \neq \emptyset$ is a finite reduced word not starting with $g^{ \pm 1}$, then $v \in\left\{w_{i}\right\}_{i}$ contradicting our first observation. If there is $g^{-m} \in\left\{w_{i}\right\}_{i}$ for some $m \geq 1$, then $g^{-1} \in\left\{w_{i}\right\}_{i}$, hence $g \in\left\{w_{i}\right\}_{i}$. This again contradicts our first observation. Therefore, the only possibility is $\left\{w_{i}\right\}_{i}=\left\{\partial \mathbb{F}_{r}\right\}$.

Hence $f=1 \otimes \tilde{f}$ for some $\tilde{f} \in C(Y, \mathbb{Z}) \otimes \mathbb{C}$. So $1 \otimes z \tilde{f}=z f=\phi(f)=1 \otimes \alpha(\tilde{f})$. Hence it follows that $z \tilde{f}=\alpha(\tilde{f})$. This shows that $z \in E_{\alpha}$. Since $z \in E_{\phi}$ was arbitrary, we obtain $E_{\phi} \subseteq E_{\alpha}$. The reverse inclusion is obvious.

We are now ready for the

Proof of Theorem [3.6. By [25, Theorem 5.10 and Proposition 5.2], $\gamma \sim_{\text {coe }} \delta$. So we just have to show $\gamma \chi_{\text {conj }} \delta$. Assume that there exists a homeomorphism $\varphi: \partial \mathbb{F}_{r} \times\left(\mathbb{Z} / M_{1}\right) \times\left(\mathbb{Z} / M_{2}\right) \cong$ $\partial \mathbb{F}_{r} \times\left(\mathbb{Z} / N_{1}\right) \times\left(\mathbb{Z} / N_{2}\right)$ and a group isomorphism $\rho: \mathbb{F}_{r} \cong \mathbb{F}_{r}$ such that $\varphi \circ \gamma_{a}=\delta_{\rho(a)} \circ \varphi$ for all $a \in \mathbb{F}_{r}$. Let $a:=a_{1}$ and $g:=\rho\left(a_{1}\right)$. Then in particular, $\varphi \circ \gamma_{a}=\delta_{g} \circ \varphi$, so that $\gamma_{a}$ and $\delta_{g}$ are conjugate, and hence $E\left(\gamma_{a}\right)=E\left(\delta_{g}\right)$.

By construction, there are $k, l \in \mathbb{Z}$ with $\delta_{g}=\beta_{g} \times \alpha_{N_{1}}^{k} \times \alpha_{N_{2}}^{l}$, where $N_{1}=p^{\infty}$ and $N_{2}=n \cdot q^{\infty}$. Proposition 3.7 yields $E\left(\delta_{g}\right)=E\left(\alpha_{N_{1}}^{k} \times \alpha_{N_{2}}^{l}\right)$. For a supernatural number $M$, let $\mathbb{T}(M)=$ $\mathbb{Z}\left[M^{-1}\right] / \mathbb{Z} \subseteq \mathbb{Q} / \mathbb{Z} \subseteq \mathbb{R} / \mathbb{Z} \cong \mathbb{T}$. If $l=0$, then $E\left(\delta_{g}\right)=E\left(\alpha_{N_{1}}^{k} \times\right.$ id $)=E\left(\alpha_{N_{1}}^{k}\right)=E\left(\alpha_{p^{\infty}}^{k}\right)$ is equal to $\{1\}$ or $\mathbb{T}\left(p^{\infty}\right)$. If $l \neq 0$, then $\mathbb{T}\left(q^{\infty}\right)=E\left(\alpha_{q^{\infty}}^{l}\right) \subseteq E\left(\alpha_{n \cdot q^{\infty}}^{l}\right) \subseteq E\left(\alpha_{p^{\infty}}^{k} \times \alpha_{n \cdot q^{\infty}}^{l}\right)=$ $E\left(\alpha_{N_{1}}^{k} \times \alpha_{N_{2}}^{l}\right)=E\left(\delta_{g}\right)$. However, $E\left(\gamma_{a}\right)=E\left(\alpha_{M_{1}} \times \mathrm{id}\right)=E\left(\alpha_{n \cdot p^{\infty}}\right)=\mathbb{T}\left(n \cdot p^{\infty}\right)$ is not equal to $\{1\}$ nor $\mathbb{T}\left(p^{\infty}\right)$ and does not contain $\mathbb{T}\left(q^{\infty}\right)$. Hence $E\left(\gamma_{a}\right) \neq E\left(\delta_{g}\right)$. This is a contradiction.

\section{From CONTINUOUS COCYCLE RIGIDITY TO CONTINUOUS ORBIT EQUIVALENCE RIGIDITY}

We introduce the notion of continuous cocycle rigidity. Let $G \curvearrowright X$ be a topological dynamical system and let $H$ be a group.

Definition 4.1. A continuous $H$-cocycle for $G \curvearrowright X$ is a continuous map $a: G \times X \rightarrow H$ such that $a\left(g_{1} g_{2}, x\right)=a\left(g_{1}, g_{2} . x\right) a\left(g_{2}, x\right)$ for all $g_{1}, g_{2} \in G, x \in X$.

In other words, $a: G \times X \rightarrow H$ is a groupoid homomorphism, where we view $G \times X$ as a groupoid by identifying it with the transformation groupoid $G \ltimes X$ attached to $G \curvearrowright X$, and view $H$ as the groupoid whose unit space is a point.

Definition 4.2. Continuous $H$-cocycles a and $a^{\prime}$ for $G \curvearrowright X$ are continuously cohomologous $\left(a \sim a^{\prime}\right)$ if there exists a continuous map $u: X \rightarrow H$ such that $a(g, x)=u(g . x) a^{\prime}(g, x) u(x)^{-1}$ for all $g \in G$ and $x \in X$. 
Definition 4.3. $G \curvearrowright X$ is continuous $H$-cocycle rigid if for every continuous $H$-cocycle a for $G \curvearrowright X$, there exists a group homomorphism $\rho: G \rightarrow H$ such that $a \sim \rho$.

Here we view $\rho$ as the cocycle $G \times X \rightarrow H,(g, x) \mapsto \rho(g)$.

The following observation provides a first link between continuous cocycle rigidity and continuous orbit equivalence rigidity.

Proposition 4.4. Let $G \curvearrowright X$ and $H \curvearrowright Y$ be topologically free systems. Assume that $G \curvearrowright$ $X \sim_{c o e} H \curvearrowright Y$, and let $\varphi, \psi, a$ and $b$ be as in Definition 2.5. If there exists a continuous map $u: X \rightarrow H$ and a group isomorphism $\rho: G \rightarrow H$ such that $a(g, x)=u(g \cdot x) \rho(g) u(x)^{-1}$ for all $g \in G, x \in X$, then ${ }_{u} \varphi: X \rightarrow Y, x \mapsto u(x)^{-1} \cdot \varphi(x)$ and $\rho$ give rise to a conjugacy between $G \curvearrowright X$ and $H \curvearrowright Y$.

Proof. ${ }_{u} \varphi$ is obviously continuous, and an easy computation shows that ${ }_{u} \varphi(g \cdot x)=\rho(g) \cdot{ }_{u} \varphi(x)$ for all $g \in G, x \in X$. It remains to show that ${ }_{u} \varphi$ is a homeomorphism.

Let $\sigma=\rho^{-1}$, define $v: Y \rightarrow G, y \mapsto(\sigma(u(\psi(y))))^{-1}$ and $\tilde{b}: H \times Y \rightarrow G,(h, y) \mapsto$ $v(h . y) \sigma(h) v(y)^{-1}$. Since

$$
\begin{aligned}
& \tilde{b}(a(g, x), \varphi(x))=v(a(g, x) \cdot \varphi(x)) \sigma(a(g, x)) v(\varphi(x))^{-1}=v(\varphi(g . x)) \sigma(a(g, x)) v(\varphi(x))^{-1} \\
= & \left.\sigma(u(g \cdot x))^{-1} \sigma(a(g, x)) \sigma(u(x))=\sigma(u(g \cdot x))^{-1} a(g, x) u(x)\right)=\sigma(\rho(g))=g,
\end{aligned}
$$

Lemma 2.10 implies that $b=\tilde{b}$. Set ${ }_{v} \psi: Y \rightarrow X, y \mapsto v(y)^{-1} \psi(y){ }_{v} \psi$ is obviously continuous, and an easy computation shows that ${ }_{v} \psi(h . y)=\rho(h){ }_{\cdot} \psi(y)$ for all $h \in H, y \in Y$. Moreover,

$$
\begin{aligned}
{ }_{v} \psi\left({ }_{u} \varphi(x)\right) & ={ }_{v} \psi\left(u(x)^{-1} \cdot \varphi(x)\right)=\sigma\left(u(x)^{-1}\right) \cdot{ }_{v} \psi(\varphi(x)) \\
& =\sigma(u(x))^{-1} v(\varphi(x))^{-1} \cdot x=\sigma(u(x))^{-1} \sigma(u(x)) \cdot x=x, \\
\text { and }{ }_{u} \varphi\left({ }_{v} \psi(y)\right) & ={ }_{u} \varphi\left(v(y)^{-1} \cdot \psi(y)\right)=\rho\left(v(y)^{-1}\right)_{u} \varphi(\psi(y)) \\
& =\rho(v(y))^{-1} u\left((\psi(y))^{-1} \cdot y=u(\psi(y)) u\left((\psi(y))^{-1} \cdot y=y .\right.\right.
\end{aligned}
$$

Thus ${ }_{u} \varphi$ is a homeomorphism, with inverse ${ }_{v} \psi$, and the proof is complete.

Continuous cocycle rigidity means that every cocycle, whether or not if comes from a continuous orbit equivalence, is continuously cohomologous to a group homomorphism. At the same time, the preceding proposition shows that for continuous orbit equivalence rigidity, cocycles are required to be continuously cohomologous to group isomorphisms. Therefore, there does not seem to be any obvious connection between continuous cocycle rigidity and continuous orbit equivalence rigidity. However, we have the following

Theorem 4.5. Suppose that $G$ is amenable and torsion-free. Let $G \curvearrowright X$ and $H \curvearrowright Y$ be topologically free systems on compact spaces $X$ and $Y$. Assume that $G \curvearrowright X \sim_{\text {coe }} H \curvearrowright Y$, and let $a: G \times X \rightarrow H$ be as in Definition 2.5. If $a \sim \rho$ for some group homomorphism $\rho: G \rightarrow H$, then $\rho$ must be an isomorphism. 
Proof. Let $u: X \rightarrow H$ be continuous such that $a(g, x)=u(g . x) \rho(g) u(x)^{-1}$ for all $g \in G$, $x \in X$. Take $x \in X$ with $G_{x}=\{e\}$. Then by Lemma 2.11, $a_{x}: G \rightarrow H, g \mapsto a(g, x)$ is bijective. Let $u_{x}: G \rightarrow H, g \mapsto u(g \cdot x)$. Then $a_{x}(g)=u_{x}(g) \rho(g) u_{x}(e)^{-1} \cdot u$ is continuous and $X$ is compact, hence $u(X) \subseteq H$ is finite. In particular, $u_{x}(G)$ is finite. Therefore, for every $g \in \operatorname{ker}(\rho), a_{x}(g) \in u_{x}(G) u_{x}(e)^{-1}$. It follows that $a_{x}(\operatorname{ker}(\rho))$ is finite. Since $a_{x}$ is injective, $\operatorname{ker}(\rho)$ is finite. But $G$ is torsion-free. This implies $\operatorname{ker}(\rho)=\{e\}$, so that $\rho$ is injective.

It remains to prove surjectivity. Since $a_{x}$ is surjective, we have $H=u(X) \rho(G) u(x)^{-1}=$ $u(X) \rho(G)$. Thus, $[H: \rho(G)]<\infty$. In particular, $H$ is also amenable. Without loss of generality, we may assume $u_{x}(e)=u(x)=e$. Otherwise, replace $\rho$ by $u(x) \rho u(x)^{-1}$ and $u_{x}$ by $u_{x} \cdot u(x)^{-1}$. Suppose that $\rho(G) \subsetneq H$. Let $R$ be a complete system of left coset representatives of $\rho(G)$ in $H$. Since $H$ is amenable, there exists a finite subset $F$ of $H$ such that $|r F \triangle F|<\frac{1}{3}|F|$ for all $r \in R$ and $|s F \triangle F|<\frac{1}{3|u(X)|}|F|$ for all $s \in u(X)$.

Assume that $|F \cap \rho(G)|>\frac{2}{3}|F|$. By assumption $(\rho(G) \subsetneq H)$, there exists $r \in R$ with $r \rho(G) \cap$ $\rho(G)=\emptyset$. So $r(F \cap \rho(G)) \cap(F \cap \rho(G))=\emptyset$, and we obtain $|r(F \cap \rho(G)) \cap F|<\frac{1}{3}|F|$. Moreover, $|r F \backslash r(F \cap \rho(G))|=|F \backslash(F \cap \rho(G))|<\frac{1}{3}|F|$. Therefore, $\frac{2}{3}|F|<|r F \cap F|=$ $|(r F \backslash r(F \cap \rho(G))) \cup(r(F \cap \rho(G)) \cap F)|<\frac{1}{3}|F|+\frac{1}{3}|F|=\frac{2}{3}|F|$. But this is a contradiction. Therefore, we must have $|F \cap \rho(G)| \leq \frac{2}{3}|F|$.

We certainly have $a_{x}^{-1}(F) \subseteq \rho^{-1}\left(\bigcup_{s \in u(X)} s^{-1} F\right)$. Hence

$$
\begin{aligned}
|F| & =\left|a_{x}^{-1}(F)\right| \leq\left|\rho^{-1}\left(\bigcup_{s \in u(X)} s^{-1} F\right)\right| \leq\left|\rho^{-1}\left(F \cup\left(\bigcup_{s \in u(X)} s^{-1} F\right) \backslash F\right)\right| \\
& \leq\left|\rho^{-1}(F)\right|+\sum_{s \in u(X)}\left|s^{-1} F \backslash F\right|<|F \cap \rho(G)|+\frac{1}{3}|F| \leq|F| .
\end{aligned}
$$

This is a contradiction. We conclude that $\rho(G)=H$.

Clearly, Proposition 4.4 and Theorem 4.5 imply

Theorem (Theorem [1.6). Let $G$ be a torsion-free amenable group. Assume that $G \curvearrowright X$ and $H \curvearrowright Y$ are topologically free systems on compact spaces $X$ and $Y$, and suppose that $G \curvearrowright X$ and $H \curvearrowright Y$ are continuously orbit equivalent. If $G \curvearrowright X$ is continuous $H$-cocycle rigid, then $G \curvearrowright X$ and $H \curvearrowright Y$ must be conjugate.

\section{Continuous COCYCle Rigidity Via GROup COHOMOlOGY}

The first goal of this section is to rephrase continuous cocycle rigidity in the language of nonabelian group cohomology. For the sake of completeness, we briefly recall the definition of non-abelian group cohomology $\left(H^{1}\right)$. We refer the reader to [21, Part Three, Appendix "Nonabelian cohomology"] and [22, Chapter I, § 5] for details. 
Let $G$ be a group acting on a group $A$ by automorphisms, denoted by $G \times A \rightarrow A,(s, a) \mapsto s . a$. A 1-cocycle of $G$ in $A$ is a map $G \rightarrow A, s \mapsto a_{s}$ such that $a_{s t}=a_{s} s$. $a_{t}$. We write $Z^{1}(G, A)$ for the set of all these 1-cocycles. Given 1-cocycles $a$ and $a^{\prime}$ of $G$ in $A$, we say that $a$ is cohomologous to $a^{\prime}\left(a \sim a^{\prime}\right)$ if there exists $b \in A$ with $a_{s}^{\prime}=b^{-1} a_{s} s . b$ for all $s \in G$. We define $H^{1}(G, A):=Z^{1}(G, A) / \sim$. Clearly, $H^{1}(G, A)$ is (covariantly) functorial in $A$.

Proposition 5.1. Let $G \curvearrowright X$ be a topological dynamical system on a compact space $X$. Let $H$ be a group.

$G \curvearrowright X$ is continuous $H$-cocycle rigid if and only if the canonical map $H \rightarrow C(X, H)$ (the map dual to $X \rightarrow\{\mathrm{pt}\})$ induces a surjective map $H^{1}(G, H) \rightarrow H^{1}(G, C(X, H))$.

Note that we equip $H$ with the trivial $G$-action, and $G$ acts on $C(X, H)$ via $(s . a)(x)=a\left(s^{-1} \cdot x\right)$.

Proof. Just check that $c: C(G, C(X, H)) \rightarrow C(G \times X, H)$ defined by $c(a)(g, x)=a_{g}(g, x)$ is a bijection, with inverse given by $c^{-1}(b)_{s}(x)=b\left(s, s^{-1} \cdot x\right)$. $c$ identifies $Z^{1}(G, C(X, H))$ with the set of continuous $H$-cocycles for $G \curvearrowright X$ in the sense of Definition 4.1. In addition, $a \sim a^{\prime}$ if and only if $c(a) \sim c\left(a^{\prime}\right)$ in the sense of Definition 4.2. It is then easy to see that for $a \in Z^{1}(G, C(X, H))$, the class $[a] \in H^{1}(G, C(X, H))$ lies in the image of the canonical map $H^{1}(G, H) \rightarrow H^{1}(G, C(X, H))$ if and only if $c(a) \sim \rho$ for some group homomorphism $\rho: G \rightarrow H$.

Using the language of non-abelian group cohomology, we now prove a positive result in continuous cocycle rigidity.

Definition 5.2. A topological dynamical system $G \curvearrowright X$ on a compact space $X$ is called almost $\mathbb{Z} G$-projective if $C(X, \mathbb{Z}) \cong \mathbb{Z} \oplus P$ as $\mathbb{Z} G$-modules, where the copy of $\mathbb{Z}$ is given by the constant functions on $X$ and $P$ is a projective $\mathbb{Z} G$-module.

We call $G \curvearrowright X$ almost $\mathbb{Z} G$-free if $P$ can be chosen to be $\mathbb{Z} G$-free.

Clearly, if a system is almost $\mathbb{Z} G$-free, then it is almost $\mathbb{Z} G$-projective.

Remark 5.3. It is easy to see that $G \curvearrowright X$ is almost $\mathbb{Z} G$-free if we can find a $\mathbb{Z}$-basis $\mathcal{B}$ for $C(X, \mathbb{Z})$ with the following properties:

- $\mathcal{B}$ is $G$-invariant,

- $1_{X} \in \mathcal{B}$,

- $G$ acts freely on $\mathcal{B} \backslash\left\{1_{X}\right\}$.

Topological Bernoulli actions for torsion-free groups turn out to be almost $\mathbb{Z} G$-free.

Example 5.4. Let $G$ be a torsion-free group and $X_{0}$ a compact space. Then the Bernoulli action $G \curvearrowright X_{0}^{G}$ is almost $\mathbb{Z} G$-free. Namely, choose a $\mathbb{Z}$-basis $\mathcal{B}_{0}$ for $C\left(X_{0}, \mathbb{Z}\right)$ with $1_{X_{0}} \in \mathcal{B}_{0}$. 
This is always possible, see for instance [3, Proposition 2.12]. Then set

$$
\mathcal{B}=\left\{\left(\bigotimes_{f \in F} b_{f}\right) \otimes 1_{X_{0}^{G \backslash F}}: F \subseteq G \text { finite, } b_{f} \in \mathcal{B}_{0}\right\} .
$$

$\mathcal{B}$ is a $\mathbb{Z}$-basis as $C\left(X_{0}^{G}, \mathbb{Z}\right)=\bigcup_{F \subseteq G \text { finite }}\left(\bigotimes_{f \in F} C\left(X_{0}\right)\right) \otimes 1_{X_{0}^{G \backslash F}}$. Obviously, $\left\{1_{X_{0}^{G}}\right\}$ lies in $\mathcal{B}$. Moreover, $G$ acts freely on $\mathcal{B} \backslash\left\{1_{X_{0}^{G}}\right\}$ as $G$ is torsion-free.

Building on the previous example, we now show that for torsion-free groups, subshifts of full shifts over finite alphabets whose forbidden words avoid a fixed letter are almost $\mathbb{Z} G$-free.

Example 5.5. Let $G$ be a torsion-free group, $A=\{0, \ldots, N\}$ a finite alphabet and $G \curvearrowright A^{G}$ the full shift. Elements in $A^{G}$ are of the form $x=\left(x_{\gamma}\right)_{\gamma \in G}$, and $g \in G$ acts by $(g . x)_{\gamma}=x_{g^{-1} \gamma}$. For every $G$-invariant closed subset $X$ of $A^{G}$ we can find a collection $\left\{F_{i}\right\}_{i \in I}$ of non-empty finite subsets of $G$ and $x_{i} \in A^{F_{i}}, i \in I$, such that

$$
X=\left\{x=\left(x_{\gamma}\right)_{\gamma} \in A^{G} \text { : For every } i \in I \text { and } g \in G, \pi_{F_{i}}(g . x) \neq x_{i}\right\} .
$$

Here $\pi_{F_{i}}$ is the canonical projection $A^{G} \rightarrow A^{F_{i}} .\left\{x_{i}\right\}_{i \in I}$ are called the forbidden words for $X$.

Now assume that $X$ is a $G$-invariant closed subset whose forbidden words $x_{i}$ satisfy $x_{i} \in$ $\{1, \ldots, N\}$, i.e., all the forbidden words avoid a fixed letter ( 0 in our case). If that is the case, then we claim that $G \curvearrowright X$ is almost $\mathbb{Z} G$-free.

Here is the reason: Obviously, $\mathcal{B}_{0}=\left\{1_{A}, 1_{\{1\}}, \ldots, 1_{\{N\}}\right\}$ is a $\mathbb{Z}$-basis for $C(A, \mathbb{Z})$. Given a finite subset $\emptyset \neq F \subseteq G$ and $x=\left(x_{f}\right)_{f \in F} \in\{1, \ldots, N\}^{F}$, let $b(F, x)=\bigotimes_{f \in F} 1_{\left\{x_{f}\right\}} \otimes 1_{A^{G \backslash F}}$. As we have seen in Example [5.4, $\mathcal{B}=\left\{1_{A^{G}}\right\} \cup\left\{b(F, x): \emptyset \neq F \subseteq G\right.$ finite, $\left.x \in\{1, \ldots, N\}^{F}\right\}$ is a $\mathbb{Z}$-basis for $C\left(A^{G}, \mathbb{Z}\right)$.

Consider the subspace $C_{0}\left(A^{G} \backslash X, \mathbb{Z}\right) \subseteq C\left(A^{G}, \mathbb{Z}\right)$ of functions vanishing on $X$. An element $\sum z_{F, x} b(F, x) \in C\left(A^{G}, \mathbb{Z}\right)\left(z_{F, x} \in \mathbb{Z}\right)$ lies in $C_{0}\left(A^{G} \backslash X, \mathbb{Z}\right)$ if and only if for every $(F, x)$ with $z_{F, x} \neq 0$, there exists $i \in I$ and $g \in G$ with $F_{i} \subseteq g F$ and $\pi_{F_{i}}(g \cdot x)=x_{i}$. Clearly, if $(F, x)$ satisfies this property, then $b(F, x)$ lies in $C_{0}\left(A^{G} \backslash X, \mathbb{Z}\right)$. Conversely, suppose that $\sum z_{F, x} b(F, x)$ lies in $C_{0}\left(A^{G} \backslash X, \mathbb{Z}\right)$ but there exists $(\tilde{F}, \tilde{x})$ with $z_{\tilde{F}, \tilde{x}} \neq 0$ such that for all $i \in I$ and $g \in G, F_{i} \nsubseteq g \tilde{F}$ or $\pi_{F_{i}}(g . \tilde{x}) \neq x_{i}$. Among all the $(\tilde{F}, \tilde{x})$ with this property, choose a pair such that $\tilde{F}$ is minimal. Define $w \in A^{G}$ by setting $w_{\gamma}=\tilde{x}_{\gamma}$ if $\gamma \in \tilde{F}$ and $w_{\gamma}=0$ otherwise. Then $w \in X$, and by our choice of $w$ and $(\tilde{F}, \tilde{x})$, we have $b(\tilde{F}, \tilde{x})(w)=1$ and $b\left(F^{\prime}, x^{\prime}\right)(w)=0$ for all $\left(F^{\prime}, x^{\prime}\right) \neq(\tilde{F}, \tilde{x})$ with $z_{F^{\prime}, x^{\prime}} \neq 0$. Hence $\left(\sum z_{F, x} b(F, x)\right)(w)=z_{\tilde{F}, \tilde{x}}$, which contradicts that $\sum z_{F, x} b(F, x)$ vanishes on $X$. This shows that

$$
\mathcal{B}_{v}:=\left\{b(F, x): \text { There is } i \in I \text { and } g \in G \text { with } F_{i} \subseteq g F \text { and } \pi_{F_{i}}(g . x)=x_{i}\right\}
$$

is a $\mathbb{Z}$-basis for $C_{0}\left(A^{G} \backslash X, \mathbb{Z}\right)$.

The canonical homomorphisms give rise to the exact sequence $0 \rightarrow C_{0}\left(A^{G} \backslash X, \mathbb{Z}\right) \rightarrow$ $C\left(A^{G}, \mathbb{Z}\right) \rightarrow C(X, \mathbb{Z}) \rightarrow 0$. One way to see this would be to apply K-theory to the exact sequence $0 \rightarrow C_{0}\left(A^{G} \backslash X\right) \rightarrow C\left(A^{G}\right) \rightarrow C(X) \rightarrow 0$. Therefore, the image $\mathcal{B}_{X}$ of $\mathcal{B} \backslash \mathcal{B}_{v}$ under 
the canonical projection $C\left(A^{G}, \mathbb{Z}\right) \rightarrow C(X, \mathbb{Z})$ is a $\mathbb{Z}$-basis for $C(X, \mathbb{Z})$. As $\mathcal{B}_{v}$ is clearly $G$ invariant, so is $\mathcal{B}_{X}$. Moreover, $1_{X} \in \mathcal{B}_{X}$, and $G$ acts freely on $\mathcal{B}_{X} \backslash\left\{1_{X}\right\} \cong \mathcal{B} \backslash\left(\mathcal{B}_{v} \cup\left\{1_{A^{G}}\right\}\right)$. Therefore, $G \curvearrowright X$ is almost $\mathbb{Z} G$-free.

The systems in Example 5.4 and Example 5.5 are not minimal. Here are examples of minimal topological dynamical systems which are almost $\mathbb{Z} G$-free.

Example 5.6. A Denjoy homeomorphism is a homeomorphism $\varphi$ of the circle which has no periodic points and is not conjugate to a rigid rotation (see for instance [18]). It turns out that there is a unique closed $\varphi$-invariant subspace $\Sigma$ of the circle which is minimal for $\varphi$. $\Sigma$ is a Cantor set. The restriction of $\varphi$ to $\Sigma$ gives rise to a Cantor minimal system $\mathbb{Z} \curvearrowright \Sigma$. In the proof of $[18$, Lemma 6.1$], K_{0}(C(\Sigma)) \cong C(\Sigma, \mathbb{Z})$ is identified as a $\mathbb{Z}[\mathbb{Z}]$-module with $\mathbb{Z} \oplus F$, where $F$ is $\mathbb{Z}[\mathbb{Z}]$-free. Hence $\mathbb{Z} \stackrel{\varphi}{\curvearrowright} \Sigma$ is almost $\mathbb{Z}[\mathbb{Z}]$-free.

It would be interesting to find more examples of almost $\mathbb{Z} G$-projective systems.

Here is why we are interested in the property "almost $\mathbb{Z} G$-projective":

Proposition 5.7. Let $G \curvearrowright X$ be a topological dynamical system on a compact space $X$, and suppose that $G \curvearrowright X$ is almost $\mathbb{Z} G$-projective. Let $H$ be an abelian $G$-group. Then the canonical map $H \rightarrow C(X, H)$ induces injections $H^{i}(G, H) \hookrightarrow H^{i}(G, C(X, H))$ for every $i \geq 0$. Moreover, assume that for every $G$-module $M, H^{1}(G, \mathbb{Z} G \otimes M) \cong\{0\}$. Then $G \curvearrowright X$ is continuous $H$ cocycle rigid.

Proof. By assumption, $C(X, \mathbb{Z}) \cong \mathbb{Z} \oplus P$ (as $\mathbb{Z} G$-modules). Then $C(X, H) \cong C(X, \mathbb{Z}) \otimes H \cong$ $H \oplus(P \otimes H)$. Therefore, for every $i \geq 0, C(G, H) \rightarrow C(C, C(X, H))$ induces injective maps $H^{i}(G, H) \hookrightarrow H^{i}(G, C(X, H))$ as these maps correspond to the canonical inclusions $H^{i}(G, H) \hookrightarrow$ $H^{i}(G, H) \oplus H^{i}(G, P \otimes H)$ under the identification $H^{i}(G, C(X, H)) \cong H^{i}(G, H) \oplus H^{i}(G, P \otimes H)$. Moreover, it is clear that for $i=1, H^{1}(G, H) \rightarrow H^{1}(G, C(X, H))$ is surjective if and only if $H^{1}(G, P \otimes H) \cong\{0\}$. But since $P$ is a projective $\mathbb{Z} G$-module, we can find a $\mathbb{Z} G$-module $Q$ such that $P \oplus Q$ is a free $\mathbb{Z} G$-module. By assumption, $H^{1}(G,(P \oplus Q) \otimes H)$ vanishes, since $(P \oplus Q) \otimes H$ is of the form $\mathbb{Z} G \otimes M$ for some $G$-module $M$. Hence $H^{1}(G, P \otimes H) \cong\{0\}$.

Lemma 5.8. Suppose that $1 \rightarrow H^{\prime} \stackrel{\iota}{\longrightarrow} H \stackrel{\pi}{\longrightarrow} H^{\prime \prime} \rightarrow 1$ is an exact sequence of groups, and assume that $H^{\prime}$ is abelian. Let $G \curvearrowright X$ be a topological dynamical system on a compact space $X$, and suppose that $G \curvearrowright X$ is almost $\mathbb{Z} G$-projective and that $H^{1}(G, \mathbb{Z} G \otimes M) \cong\{0\}$ for every $G$-module $M$.

If $G \curvearrowright X$ is continuous $H^{\prime \prime}$-cocycle rigid, then $G \curvearrowright X$ is continuous $H$-cocycle rigid.

Proof. Write $C^{\prime}=C\left(X, H^{\prime}\right), C=C(X, H)$ and $C^{\prime \prime}=C\left(X, H^{\prime \prime}\right)$. Let $i: C^{\prime} \rightarrow C$ and $p: C \rightarrow C^{\prime \prime}$ be the homomorphisms induced by $\iota$ and $\pi$. We get the following commutative 
diagram with exact rows:

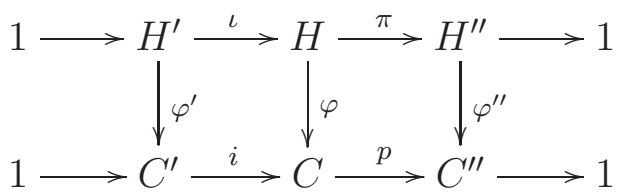

where $\varphi^{\prime}, \varphi$ and $\varphi^{\prime \prime}$ are the canonical homomorphisms.

Take $x \in H^{1}(G, C)$. Since $\varphi_{*}^{\prime \prime}$ is surjective, we can find $\xi^{\prime \prime} \in H^{1}\left(G, H^{\prime \prime}\right)$ with $\varphi_{*}^{\prime \prime}\left(\xi^{\prime \prime}\right)=p_{*}(x)$. Let us first prove the following

Claim: There exists $\zeta \in H^{1}(G, H)$ with $\pi_{*}(\zeta)=\xi^{\prime \prime}$.

Proof of the claim: Let $\lambda^{\prime \prime}$ be a 1-cocycle of $G$ in $H^{\prime \prime}$ representing $\xi^{\prime \prime}$. Lift $\lambda^{\prime \prime}$ to a map $\lambda: G \rightarrow H$ such that $\pi \circ \lambda=\lambda^{\prime \prime}$. Then, as in [22, Chapter I, $\left.\S 5.6\right]$, define a 2-cocycle $\lambda^{\prime}$ of $G$ in $H^{\prime}$ by setting $\lambda_{s, t}^{\prime}:=\lambda_{s} s . \lambda_{t} \lambda_{s t}^{-1}$ and let $\Delta\left(\lambda^{\prime \prime}\right):=\left[\lambda^{\prime}\right] \in H^{2}\left(G, H^{\prime}\right)$. Since $p \circ \varphi \circ \lambda=$ $\varphi^{\prime \prime} \circ \pi \circ \lambda=\varphi^{\prime \prime} \circ \lambda^{\prime \prime}, \varphi \circ \lambda$ is a lift of $\varphi \circ \lambda$. Moreover, $\left[\varphi^{\prime \prime} \circ \lambda^{\prime \prime}\right]=\varphi_{*}^{\prime \prime}\left[\lambda^{\prime \prime}\right]=\varphi_{*}^{\prime \prime}\left(\xi^{\prime \prime}\right)=p_{*}(x)$ lies in $\operatorname{Im}\left(p_{*}\right)$. Therefore, by [22, Chapter I, $\S 5.6$, Proposition 41], $\Delta\left(\varphi^{\prime \prime} \circ \lambda^{\prime \prime}\right)=0$ in $H^{2}\left(G, C^{\prime}\right)$. Hence $0=\Delta\left(\varphi^{\prime \prime} \circ \lambda^{\prime \prime}\right)=\left[\varphi^{\prime} \circ \lambda^{\prime}\right]=\varphi_{*}^{\prime}\left[\lambda^{\prime}\right]=\varphi_{*}^{\prime}\left(\Delta\left(\lambda^{\prime \prime}\right)\right)$. As $\varphi_{*}^{\prime}$ is injective by Proposition 5.7 , we obtain $\Delta\left(\lambda^{\prime}\right)=0$. And thus, again by [22, Chapter I, $\S 5.6$, Proposition 41], $\xi^{\prime \prime} \operatorname{lies}$ in $\operatorname{Im}\left(\pi_{*}\right)$. This proves our claim.

So we can find $\zeta \in H^{1}(G, H)$ with $\pi_{*}(\zeta)=\xi^{\prime \prime}$. Then $p_{*}\left(\varphi_{*}(\zeta)\right)=\varphi_{*}^{\prime \prime}\left(\pi_{*}(\zeta)\right)=\varphi_{*}^{\prime \prime}\left(\xi^{\prime \prime}\right)=p_{*}(x)$. Let $\beta$ be a 1-cocycle of $G$ in $H$ representing $\zeta$. Let $b=\varphi(\beta)$. Then $\varphi_{*}(\zeta)=[b]$. Twisting by $b$ gives rise to a commutative diagram

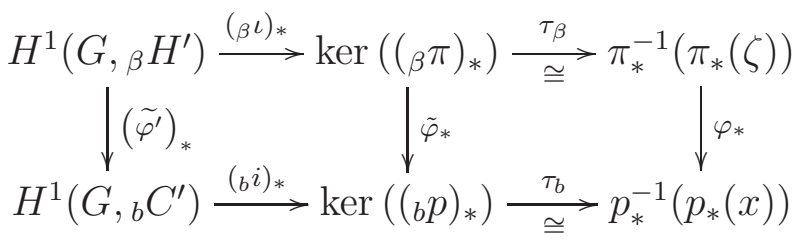

Twisting and the bijections $\tau_{b}$ are explained in [22, Chapter I, $\left.\S 5.3\right]$ and at the beginning of [22, Chapter I, $\S 5.4]$. By [22, Chapter I, § 5.5, Proposition 38], $\left({ }_{b} i\right)_{*}: H^{1}\left(G,{ }_{b} C\right) \rightarrow \operatorname{ker}\left(\left({ }_{b} p\right)_{*}\right)$ is surjective. Moreover, $\left(\tilde{\varphi^{\prime}}\right)_{*}: H^{1}\left(G,{ }_{\beta} H^{\prime}\right) \rightarrow H^{1}\left(G,{ }_{b} C^{\prime}\right)$ is surjective by Proposition 5.7. Hence, by commutativity of the left square in (5), $\tilde{\varphi}_{*}: \operatorname{ker}\left(\left({ }_{\beta} \pi\right)_{*}\right) \rightarrow \operatorname{ker}\left(\left({ }_{b} p\right)_{*}\right)$ is surjective. Commutativity of the right square in (5) implies that $\varphi_{*}: \pi_{*}^{-1}\left(\pi_{*}(\zeta)\right) \rightarrow p_{*}^{-1}\left(p_{*}(x)\right)$ is surjective. In particular, there exists $\xi \in \pi_{*}^{-1}\left(\pi_{*}(\zeta)\right) \subseteq H^{1}(G, H)$ with $\varphi_{*}(\xi)=x$.

Corollary 5.9. Let $G \curvearrowright X$ be a topological dynamical system on a compact space $X$. Suppose that $G \curvearrowright X$ is almost $\mathbb{Z} G$-projective and that $H^{1}(G, \mathbb{Z} G \otimes M) \cong\{0\}$ for every $G$-module $M$.

Then $G \curvearrowright X$ is continuous $H$-cocycle rigid for every solvable group $H$.

Proof. We proceed inductively on the length of a series $\{1\}=H_{0} \subseteq H_{1} \subseteq \ldots \subseteq H_{n}=H$ with $H_{i} \triangleleft H$ for all $1 \leq i \leq n$ and $H_{i} / H_{i-1}$ abelian for all $1 \leq i \leq n$. The case $n=1$ is taken care 
of by Proposition 5.7. To go from $n-1$ to $n$, consider the series $\{1\}=H_{1} / H_{1} \subseteq H_{2} / H_{1} \subseteq$ $\ldots \subseteq H_{n} / H_{1}=H / H_{1}$. By induction hypothesis, $G \curvearrowright X$ is continuous $H / H_{1}$-cocycle rigid. Applying Lemma 5.8 to $1 \rightarrow H_{1} \rightarrow H \rightarrow H / H_{1} \rightarrow 1$, we obtain that $G \curvearrowright X$ is continuous $H$-cocycle rigid.

Remark 5.10. If $G$ is a duality group in the sense of [2, Chapter VIII, $\S 10]$ with $\operatorname{cd}(G) \neq 1$, then $H^{1}(G, \mathbb{Z} G \otimes M) \cong\{0\}$ for every $G$-module $M$.

Clearly, Corollary 5.9 and Remark 5.10 imply

Theorem (Theorem 1.7). Let $G$ be a torsion-free group, let $X$ be a compact space, and suppose that $G \curvearrowright X$ is a topological dynamical system which is almost $\mathbb{Z} G$-projective. Furthermore, assume that $G$ is a duality group in the sense of [2, Chapter VIII, $\S 10]$ with $c d(G) \neq 1$. Then $G \curvearrowright X$ is continuous $H$-cocycle rigid for every solvable group $H$.

\section{Continuous orbit couples and topological Couplings}

Let us build the bridge between continuous orbit equivalence and topological couplings. Let $G$ and $H$ be groups.

Definition 6.1. A topological coupling for $G$ and $H$ consists of a locally compact space $\Omega$ with commuting free and proper left $G$ - and right $H$-actions which admit compact open fundamental domains $\bar{X}$ (for the $H$-action) and $\bar{Y}$ (for the $G$-action).

A topological coupling is topologically free if the corresponding action $G \times H \curvearrowright \Omega$ is topologically free.

We often write $G \curvearrowright \Omega \curvearrowleft H$ for our topological coupling, or $G \curvearrowright \Omega \curvearrowleft \Omega_{\bar{Y}} H$ if we want to keep track of the fundamental domains. Here, by a fundamental domain $\bar{X}$ for $\Omega \curvearrowleft H$, we mean a subspace $\bar{X} \subseteq \Omega$ such that the inclusion $\bar{X} \hookrightarrow \Omega$ induces a homeomorphism $\bar{X} \cong \Omega / H$. Since we require $\bar{X}$ to be compact and open, this means that $\bar{X} \times H \rightarrow \Omega,(x, h) \mapsto x h$ is a homoemorphism (where $H$ carries the discrete topology). For the sake of brevity, we refer to topologically free topological couplings as topologically free couplings.

Moreover, topological couplings $G \underset{\bar{X}_{1}}{\curvearrowright} \Omega_{1} \underset{\bar{Y}_{1}}{\curvearrowleft} H$ and $G \underset{\bar{X}_{2}}{\curvearrowright} \Omega_{2} \underset{\bar{Y}_{2}}{\curvearrowleft} H$ are isomorphic if there exists a $G \times H$-equivariant homeomorphism $\Omega_{1} \cong \Omega_{2}$ sending $\bar{X}_{1}$ to $\bar{X}_{2}$ and $\bar{Y}_{1}$ to $\bar{Y}_{2}$.

We now introduce a notion which is similar to, but weaker than continuous orbit equivalence.

Definition 6.2. Let $G \curvearrowright X$ and $H \curvearrowright Y$ be topological dynamical systems.

A continuous map $p: X \rightarrow Y$ is called a continuous orbit map if there exists a continuous map $a: G \times X \rightarrow H$ such that $p(g . x)=a(g, x) \cdot p(x)$. 
$A$ continuous orbit couple for $G \curvearrowright X$ and $H \curvearrowright Y$ consists of continuous orbit maps $p: X \rightarrow Y$ and $q: Y \rightarrow X$ such that there exist continuous maps $g: X \rightarrow G$ and $h: Y \rightarrow H$ such that $q p(x)=g(x) \cdot x$ and $p q(y)=h(y) \cdot y$ for all $x \in X, y \in Y$.

$A$ continuous orbit couple for $G$ and $H$ consists of topological dynamical systems $G \curvearrowright X$ and $H \curvearrowright Y$ on compact spaces $X$ and $Y$ and a continuous orbit couple $(p, q)$ for $G \curvearrowright X$ and $H \curvearrowright Y$.

We call a continuous orbit couple for $G$ and $H$ topologically free if $G \curvearrowright X$ and $H \curvearrowright Y$ are topologically free.

Note that if $(p, q)$ is a continuous orbit couple for $G \curvearrowright X$ and $H \curvearrowright Y$ such that $q=p^{-1}$ (i.e., $g \equiv e$ and $h \equiv e)$, then $G \curvearrowright X$ and $H \curvearrowright Y$ are continuously orbit equivalent. In that case, we call $(p, q)$ a continuous orbit equivalence.

Continuous orbit couples $\left(p_{i}, q_{i}\right)$ for $G \curvearrowright X_{i}$ and $H \curvearrowright Y_{i}, i=1,2$, are isomorphic if there exist

a $G$-equivariant homeomorphism $X_{1} \stackrel{\cong}{\longrightarrow} X_{2}$ and an $H$-equivariant homeomorphism $Y_{1} \stackrel{\cong}{\longrightarrow} Y_{2}$ such that the diagrams
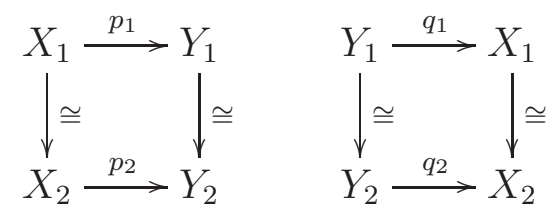

commute.

The main goal of this section is to prove the following

Theorem 6.3. Let $G$ and $H$ be groups. There is a one-to-one correspondence between isomorphism classes of topologically free couplings for $G$ and $H$ and isomorphism classes of topologically free continuous orbit couples for $G$ and $H$, with the following additional properties:

(a) topological couplings with $\bar{X}=\bar{Y}$ correspond to continuous orbit equivalences;

(b) for topologically free couplings $G \underset{\bar{X}_{1}}{\curvearrowright} \Omega_{1} \underset{\bar{X}_{1}}{\curvearrowleft} H$ and $G \underset{\bar{X}_{2}}{\curvearrowright} \Omega_{2} \underset{\bar{X}_{2}}{\curvearrowleft} H$, there exists $a G \times H$ equivariant homeomorphism $\Omega_{1} \cong \Omega_{2}$ (which might or might not preserve the fundamental domains) if and only if the cocycles $a_{1}, b_{1}, a_{2}$ and $b_{2}$ of the corresponding continuous orbit equivalence satisfy $a_{1} \sim a_{2}$ and $b_{1} \sim b_{2}$;

(c) for a topologically free coupling $G \underset{\bar{X}}{\curvearrowright} \Omega \Omega_{\bar{X}} H$, there exists an isomorphism $\rho: G \cong$ $H$ and a $G \times H$-equivariant $\operatorname{map} \stackrel{X}{\Omega} \rightarrow{ }^{X} G$ (where $G$ is equipped with the canonical left $G$-action, and the right $H$-action is given by $\rho$ ) if and only if the cocycle a (see Definition (6.2) of the corresponding continuous orbit equivalence satisfies $a \sim \rho$ for an isomorphism $\rho: G \cong H$ (the same isomorphism as for the coupling). 
For the proof of this theorem, we will now present explicit constructions of continuous orbit couples out of topological couplings and vice versa. The constructions are really the topological analogues of those in [7, §3] (see also [23] and [20]).

6.1. From topological couplings to continuous orbit couples. Suppose that we are given

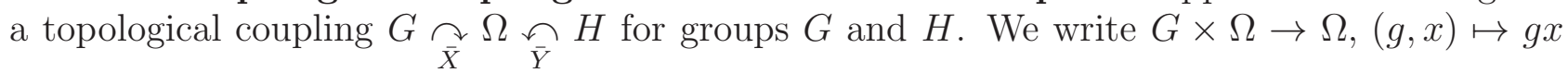
and $\Omega \times H \rightarrow \Omega,(x, h) \mapsto x h$ for the left $G$-action and right $H$-action.

Set $X:=\bar{X}$ and $Y:=\bar{Y}$. Define a map $p: X \rightarrow Y$ by requiring $G x \cap Y=\{p(x)\}$ for all $x \in X$. The intersection on the left hand side is taken in $\Omega$. Since $Y \subseteq \Omega$ is compact and open, $p$ is continuous. Moreover, by construction, there is a continuous map $\gamma: X \rightarrow G$ with $p(x)=\gamma(x) x$.

We now define a $G$-action, denoted by $G \times X \rightarrow X,(g, x) \mapsto g . x$, as follows: For every $g \in G$ and $x \in X$, there exists a unique $\alpha(g, x) \in H$ such that $g x \in X \alpha(g, x)$. Since $X$ is compact and open, $\alpha: G \times X \rightarrow H$ is continuous. Set $g \cdot x:=g x \alpha(g, x)^{-1}$. It is easy to check that this defines a (left) $G$-action on $X$.

Similarly, we define a continuous map $q: Y \rightarrow X$ by requiring $X \cap y H=\{q(y)\}$ for all $y \in Y$, and let $\eta: Y \rightarrow H$ be the continuous map satisfying $q(y)=y \eta(y)$. To define an $H$-action on $Y$, let $\beta(y, h) \in G$ be such that $y h \in \beta(y, h) Y$. Again, $\beta: Y \times H \rightarrow G$ is continuous. Set $h . y:=\beta\left(y, h^{-1}\right)^{-1} y h^{-1}$.

Let us check that $(p, q)$ is a continuous orbit couple for $G$ and $H$. To determine $p(g \cdot x)=$ $p\left(g x \alpha(g, x)^{-1}\right)$, we need to identify $\operatorname{Ggx} \alpha(g, x)^{-1} \cap Y$. We have

$$
\operatorname{Gg} x \alpha(g, x)^{-1} \ni \beta\left(\gamma(x) x, \alpha(g, x)^{-1}\right)^{-1} \gamma(x) x \alpha(g, x)^{-1} \in Y,
$$

so $p(g . x)=\beta\left(\gamma(x) x, \alpha(g, x)^{-1}\right)^{-1} \gamma(x) x \alpha(g, x)^{-1}=\alpha(g, x) \cdot(\gamma(x) x)=\alpha(g, x) \cdot p(x)$. Similarly, in order to identify $q(h . y)=q\left(\beta\left(y, h^{-1}\right)^{-1} y h^{-1}\right)$, we need to determine $X \cap \beta\left(y, h^{-1}\right)^{-1} y h^{-1} H$. As

$$
X \ni \beta\left(y, h^{-1}\right)^{-1} y \eta(y) \alpha\left(\beta\left(y, h^{-1}\right)^{-1}, y \eta(y)\right)^{-1} \in \beta\left(y, h^{-1}\right)^{-1} y h^{-1} H,
$$

we conclude that $q(y . h)=\beta\left(y, h^{-1}\right)^{-1} y \eta(y) \alpha\left(\beta\left(y, h^{-1}\right)^{-1}, y \eta(y)\right)^{-1}=\beta\left(y, h^{-1}\right)^{-1} \cdot y \eta(y)=$ $\beta\left(y, h^{-1}\right)^{-1} \cdot q(y)$. Finally, $q p(x)=q(\gamma(x) x)=\gamma(x) x \alpha(\gamma(x), x)^{-1}=\gamma(x) \cdot x$ and $p q(y)=$ $p(y \eta(y))=\beta(y, \eta(y))^{-1} y \eta(y)=\eta(y)^{-1} \cdot y$. All in all, we see that $(p, q)$ is a continuous orbit couple for $G \curvearrowright X$ and $H \curvearrowright Y$ in the sense of Definition 6.2, with $a(g, x)=\alpha(g, x)$, $b(h, y)=\beta\left(y, h^{-1}\right)^{-1}, g(x)=\gamma(x)$ and $h(y)=\eta(y)^{-1}$.

Note that our coupling does not need to be topologically free for this construction. However, it is clear that $G \curvearrowright \Omega \curvearrowleft H$ is topologically free (i.e., $G \times H \curvearrowright \Omega$ is topologically free) if and only if $G \curvearrowright X$ and $H \curvearrowright Y$ are topologically free.

6.2. From continuous orbit couples to topological couplings. Let $G \curvearrowright X$ and $H \curvearrowright Y$ be topologically free systems on compact spaces $X$ and $Y$. Assume that $(p, q)$ is a continuous orbit couple for $G \curvearrowright X$ and $H \curvearrowright Y$, and let $a, b, g$ and $h$ be as in Definition 6.2. Define 
commuting left $G$ - and right $H$-actions on $X \times H$ by $g(x, h)=(g \cdot x, a(g, x) h),(x, h) h^{\prime}=\left(x, h h^{\prime}\right)$. Furthermore, define commuting left $G$ - and right $H$-actions on $G \times Y$ by $g^{\prime}(g, y)=\left(g^{\prime} g, y\right)$ and $(g, y) h=\left(g b\left(h^{-1}, y\right)^{-1}, h^{-1} \cdot y\right)$.

A straightforward computation, using the cocycle identities (see Lemma 2.8) for $a$ and $b$, shows that $\Theta: X \times H \rightarrow G \times Y,(x, h) \mapsto\left(g(x)^{-1} b\left(h^{-1}, p(x)\right)^{-1}, h^{-1} \cdot p(x)\right)$ is a $G$ - and $H$ equivariant homeomorphism whose inverse is given by $\Theta^{-1}: G \times Y \rightarrow X \times H,(g, y) \mapsto$ $(g . q(y), a(g, q(y)) h(y))$. Therefore, if we set $\Omega=X \times H$ as a $G \times H$-space and set $\bar{X}=X \times\{e\}$, $\bar{Y}=\Theta^{-1}(\{e\} \times Y)$, then this yields the desired topologically free coupling $G \underset{\bar{X}}{\curvearrowright_{\bar{Y}}} H$.

Note that topological freeness of $G \curvearrowright X$ and $H \curvearrowright Y$ ensures that $a$ and $b$ satisfy the cocycle identities (as in Lemma 2.8), which are needed in the preceding computations.

\subsection{One-to-one correspondence and consequences. We can now finish the}

Proof of Theorem 6.3. It is straightforward to check that the constructions described in $\S 6.1$ and $\S 6.2$ are inverse to each other up to isomorphism. For instance, if we start with a topologically free coupling $G \underset{\bar{X}}{\curvearrowright} \Omega \underset{\bar{Y}}{\curvearrowleft} H$, construct a continuous orbit couple and then again a topological coupling, we end up with a coupling of the form $G \underset{\tilde{X}}{\curvearrowright} \tilde{\Omega} \underset{\tilde{Y}}{\curvearrowleft} H$ where $\tilde{\Omega}=X \times H \cong G \times Y$, $\tilde{X}=X \times\{e\}$ and $\tilde{Y} \cong\{e\} \times Y$. It is then obvious that $\tilde{\Omega}=X \times H \rightarrow \Omega,(x, h) \mapsto x h$ is an isomorphism of the couplings $G \underset{\tilde{X}}{\curvearrowright} \tilde{\Omega} \underset{\tilde{Y}}{\curvearrowleft} H$ and $G \underset{\bar{X}}{\curvearrowright} \underset{\bar{Y}}{\curvearrowleft} H$. Conversely, if we start with a continuous orbit couple $(p, q)$ for topologically free systems $G \curvearrowright X$ and $H \curvearrowright Y$, construct a topological coupling and then again a continuous orbit couple, we end up with a continuous $\operatorname{orbit}$ couple $(\tilde{p}, \tilde{q})$ for $G \curvearrowright \tilde{X}$ and $H \curvearrowright \tilde{Y}$ where $\tilde{X}=X \times\{e\}$ and $\tilde{Y} \cong\{e\} \times Y$. The canonical isomorphisms $X \cong X \times\{e\}$ and $Y \cong\{e\} \times Y$ give rise to an isomorphism between $(p, q)$ and $(\tilde{p}, \tilde{q})$.

The additional properties (a), (b) and (c) are also easy to check.

In particular, this proves Theorem 1.8 .

Corollary 6.4. Assume that there exists a topologically free continuous orbit couple for groups $G$ and $H$. If $G$ is finitely generated, then so is $H$, and $G$ and $H$ are quasi-isometric.

In particular, if topologically free systems $G \curvearrowright X$ and $H \curvearrowright Y$ on compact spaces $X$ and $Y$ are continuously orbit equivalent, and if $G$ is finitely generated, then so is $H$, and $G$ and $H$ are quasi-isometric.

Proof. By Theorem 6.3, if there exists a topologically free continuous orbit couple for $G$ and $H$, then there exists a (topologically free) topological coupling for $G$ and $H$. Our claim follows from [4, Chapter IX, Exercise 34]. 


\section{Conclusions}

Now we are ready for the proofs of Theorem 1.3 and Theorem 1.4 .

Theorem (Theorem 1.3). Let $G$ be a torsion-free group, and let $H$ be a finitely generated nilpotent group which is not virtually infinite cyclic. Assume that $G \curvearrowright X$ is a topologically free system on a compact space $X$ such that $G \curvearrowright X$ is almost $\mathbb{Z} G$-projective. Furthermore, let $H \curvearrowright Y$ be a topologically free system. If $G \curvearrowright X$ and $H \curvearrowright Y$ are continuously orbit equivalent, then they must be conjugate.

Proof of Theorem 1.3. Corollary 6.4 implies that $G$ is finitely generated, and that $G$ and $H$ are quasi-isometric. So $G$ is virtually nilpotent but not virtually infinite cyclic because $H$ has these properties (see [12]). This means that $G$ contains a finitely generated, torsion-free, nilpotent group as a subgroup of finite index. Therefore, by [2, Chapter VIII, Proposition (10.2)], $G$ has to be a duality group. Since $G$ is not virtually infinite cyclic, we know that $\operatorname{cd}(G) \neq 1$. Thus $G \curvearrowright X$ is continuous $H$-cocycle rigid by Theorem 1.7. Now assume that $G \curvearrowright X \sim_{\text {coe }} H \curvearrowright Y$. As $G$ is torsion-free and virtually nilpotent, hence amenable, and because $G \curvearrowright X$ is continuous $H$-cocycle rigid, Theorem 1.6 implies that $G \curvearrowright X \sim_{\text {conj }} H \curvearrowright Y$.

Here is our second main result, followed by its proof.

Theorem (Theorem 1.4). Let $G$ be a duality group in the sense of [2, Chapter VIII, $\S 10]$ which is not infinite cyclic, and let $H$ be a finitely generated solvable group. Assume that $G \curvearrowright X$ is a topologically free system on a compact space $X$ such that $G \curvearrowright X$ is almost $\mathbb{Z} G$-projective. Furthermore, let $H \curvearrowright Y$ be a topologically free system. If $G \curvearrowright X$ and $H \curvearrowright Y$ are continuously orbit equivalent, then they must be conjugate.

Proof of Theorem 1.4. By Corollary 6.4, $G$ is finitely generated and quasi-isometric to $H$. Therefore, $G$ is amenable (see [4, Chapter IV, 50. Geometric properties]). Moreover, as $G$ is a duality group, it is torsion-free. As $G$ is not infinite cyclic, we must have $c d(G) \neq 1$. Hence Theorem 1.7 implies that $G \curvearrowright X$ is continuous $H$-cocycle rigid. Now assume that $G \curvearrowright X \sim_{\text {coe }} H \curvearrowright Y$. Since $G$ is torsion-free and amenable, and because $G \curvearrowright X$ is continuous $H$-cocycle rigid, Theorem 1.6 implies that $G \curvearrowright X \sim_{\text {conj }} H \curvearrowright Y$.

\section{REFERENCES}

[1] M. Boyle and J. TomiYama, Bounded topological orbit equivalence and $C^{*}$-algebras, J. Math. Soc. Japan 50 (1998), 317-329.

[2] K. S. Brown, Cohomology of groups, Graduate Texts in Mathematics 87, Springer, 1982.

[3] J. Cuntz, S. Echterhoff and X. LI, On the K-theory of crossed products by automorphic semigroup actions, Quart. J. Math. 64 (2013), 747-784.

[4] P. DE LA HARPE, Topics in geometric group theory, Chicago Lectures in Mathematics, University of Chicago Press, 2000. 
[5] J. Feldman and C. Moore, Ergodic equivalence relations, cohomology, and von Neumann algebras. I, Trans. Amer. Math. Soc. 234 (1977), 289-324.

[6] J. Feldman and C. Moore, Ergodic equivalence relations, cohomology, and von Neumann algebras. II, Trans. Amer. Math. Soc. 234(1977), 325-359.

[7] A. Furman, Orbit equivalence rigidity, Ann. of Math. (2) 150 (1999), 1083-1108.

[8] D. Gaboriau, Orbit equivalence and measured group theory, in Proceedings of the ICM (Hyderabad, India, 2010), Vol. III, Hindustan Book Agency, 2010, pp. 1501-1527.

[9] T. Giordano, H. Matui, I.F. Putnam and C.F. Skau, Orbit equivalence for Cantor minimal $\mathbb{Z}^{2}$-systems, J. Amer. Math. Soc. 21 (2008), 863-892.

[10] T. Giordano, H. Matui, I.F. Putnam and C.F. Skau, Orbit equivalence for Cantor minimal $\mathbb{Z}^{d}$-systems, Invent. Math. 179 (2010), 119-158.

[11] T. Giordano, I.F. Putnam and C.F. Skau, Topological orbit equivalence and C-crossed products, J. Reine Angew. Math. 469 (1995), 51-111.

[12] M. Gromov, Groups of polynomial growth and expanding maps, Publ. Math. I.H.É.S. 53 (1981), 53-73.

[13] A. IoAnA, $W^{*}$-superrigidity for Bernoulli actions of property (T) groups, J. Amer. Math. Soc. 24 (2011), $1175-1226$.

[14] Y. KIDA, Measure equivalence rigidity of the mapping class group, Ann. of Math. (2) 171 (2010), 1851-1901.

[15] N. Monod and Y. Shalom, Orbit equivalence rigidity and bounded cohomology, Ann. of Math. (2) 164 (2006), 825-878.

[16] S. PopA, Cocycle and orbit equivalence superrigidity for malleable actions of w-rigid groups, Invent. Math. 170 (2007), 243-295.

[17] S. PopA, On the superrigidity of malleable actions with spectral gap, J. Amer. Math. Soc. 21 (2008), 981-1000.

[18] I.F. Putnam, K. Schmidt and C.F. Skau, $C^{*}$-algebras associated with Denjoy homeomorphisms of the circle, J. Operator Theory 16 (1986), 99-126.

[19] J. Renault, Cartan subalgebras in $C^{*}$-algebras, Irish Math. Soc. Bulletin 61 (2008), 29-63.

[20] R. SAuer, Homological invariants and quasi-isometry, Geom. Funct. Anal. 16 (2006), 476-515.

[21] J.P. Serre, Local Fields, Springer, Berlin Heidelberg New York, 1979.

[22] J.P. Serre, Galois Cohomology, Springer, Berlin Heidelberg New York, 1997.

[23] Y. Shalom, Harmonic analysis, cohomology, and the large-scale geometry of amenable groups, Acta Math. 192 (2004), 119-185.

[24] I.M. Singer, Automorphisms of finite factors, Amer. J. Math. 77 (1955), 117-133.

[25] Y. Suzuki, Amenable minimal Cantor systems of free groups arising from diagonal actions, arXiv: 1312.7098v4, accepted for publication in J. Reine Angew. Math..

[26] S. VAes, Rigidity results for Bernoulli actions and their von Neumann algebras (after Sorin Popa), Séminaire Bourbaki, exp. no. 961, Astérisque 311 (2007), 237-294.

[27] S. VAEs, Rigidity for von Neumann algebras and their invariants, in Proceedings of the ICM (Hyderabad, India, 2010), Vol. III, Hindustan Book Agency, 2010, pp. 1624-1650.

[28] R.J. ZimmeR, Ergodic theory and semisimple groups, Vol. 81 of Monographs in Mathematics, Birkhäuser Verlag, Basel, 1984.

Xin Li, School of Mathematical Sciences, Queen Mary University of London, Mile End Road, LONDON E1 4NS, United Kingdom

E-mail address: xin.li@qmul.ac.uk 